\title{
Interaction between mean flow and thermo-hydraulic behaviour in inclined louvered fins
}

\author{
C. T’Joen ${ }^{1,2 *}$, H. Huisseune ${ }^{1}$, H. Canière ${ }^{1}$, H.J. Steeman ${ }^{1}$, A. Willockx ${ }^{1}$ and M. De Paepe ${ }^{1}$ \\ ${ }^{1}$ Department of Flow, Heat and Combustion Mechanics, Ghent University \\ Sint-Pietersnieuwstraat 41 - 9000 Gent - Belgium \\ ${ }^{2}$ Delft University of Technology, Department Radiation, Radionuclides \& Reactors, \\ Mekelweg 15, 2629 JB Delft, The Netherlands
}

\begin{abstract}
In this study the inclined louvered fin, a hybrid fin design based on the slit fin and louvered fin design is considered. The goal of the research program is to investigate the interaction between the flow behaviour (flow deflection and transition to unsteady flow) and the thermo-hydraulics of the fin design. This approach was selected in order to reveal the flow physics behind the transitions found in the thermo-hydraulic data. Through flow visualization (dye injection in a water tunnel) the flow deflection and transition to unsteady flow was studied in different configurations and for varying Reynolds number. The flow deflection was quantified through the 'fin angle alignment factor'. Validated CFD simulations were used to further explore flow behaviour. In parallel, wind tunnel measurements were performed measuring the local heat transfer coefficients for the different louvers and the overall pressure drop. The impact of the fin pitch, fin angle and Reynolds number were studied. A comparison of both local and global parameters to the observed flow behaviour revealed the strong coupling between the flow and the thermo-hydraulics showing evidence of boundary layer driven flow.
\end{abstract}

Keywords: inclined louvered fins, flow field, thermo-hydraulics

\section{Nomenclature}

$\begin{array}{ll}A_{c} & \text { minimal free flow area }\left[\mathrm{m}^{2}\right] \\ A_{\text {ext }} & \text { exterior surface area }\left[\mathrm{m}^{2}\right] \\ \mathrm{f} & \text { Fanning friction factor [-] } \\ \mathrm{F}_{\mathrm{p}} & \text { fin pitch [m] } \\ \mathrm{G}_{\mathrm{c}} & \text { Mass flux through the minimal free flow area }\left[\mathrm{kg} / \mathrm{m}^{2} \mathrm{~s}\right] \\ \mathrm{h} & \text { convective heat transfer coefficient }\left[\mathrm{W} / \mathrm{m}^{2} \mathrm{~K}\right] \\ \mathrm{j} & \text { Colburn j-factor [-] }\end{array}$

* Corresponding author: E-mail: christophe.tjoen@ugent.be, Tel: +32 9264 33 55, Fax: +32 92643575 


\begin{tabular}{|c|c|}
\hline $\mathrm{k}$ & thermal conductivity [W/mK] \\
\hline $\mathrm{L}_{\mathrm{p}}$ & louver pitch $[\mathrm{m}]$ \\
\hline $\mathrm{Nu}$ & Nusselt number [-] \\
\hline$\Delta \mathrm{p}$ & pressure drop $[\mathrm{Pa}]$ \\
\hline $\mathrm{q}$ & surface heat flux [W/m²] \\
\hline $\mathrm{q}_{\text {cond }}$ & conductive heat flux [W/m²] \\
\hline $\operatorname{Re}$ & Reynolds number [-] \\
\hline $\mathrm{Ri}$ & Richardson number [-] \\
\hline St & Stanton number [-] \\
\hline $\mathrm{T}$ & temperature $[\mathrm{K}]$ \\
\hline $\mathrm{t}$ & fin thickness [m] \\
\hline $\mathrm{x}, \mathrm{y}, \mathrm{z}$ & spatial coordinate $[\mathrm{m}]$ \\
\hline $\mathrm{X}^{*}$ & dimensionless louver coordinate, Eq. (7) [-] \\
\hline \multicolumn{2}{|c|}{ Greek symbols } \\
\hline$\alpha$ & flow efficiency [-] \\
\hline$\zeta$ & fin angle alignment factor [-] \\
\hline$\eta$ & flow efficiency [-] \\
\hline$\theta$ & louver angle $\left[^{\circ}\right]$ \\
\hline$\rho$ & density $\left[\mathrm{kg} / \mathrm{m}^{3}\right]$ \\
\hline$\sigma$ & contraction ratio, $\mathrm{A}_{\mathrm{c}} / \mathrm{A}_{\mathrm{ext}}[-]$ \\
\hline$\varphi$ & fin angle $\left[^{\circ}\right]$ \\
\hline \multicolumn{2}{|c|}{ Subscripts } \\
\hline balsa & balsa wood \\
\hline bottom & bottom surface \\
\hline ref & reference \\
\hline surf & surface \\
\hline top & top surface \\
\hline
\end{tabular}

\section{Introduction}

Heat transfer as an energy transfer process affects every facet of our day-to-day lives, ranging from the generation of power (electricity), to cooking, preserving food (refrigeration) and providing a suitable indoor climate (HVAC - air conditioning). Because of the huge variety in the nature of the processes involving heat transfer, heat exchangers can take on many different forms (a classification can be found in e.g. Shah and Sekulic [1]). Regardless of their form, the heat exchangers are very important to the overall efficiency of the energy transfer process, and to the cost and size of the system. A very typical application is the 
exchange of heat between a liquid and a gas, mostly air (e.g. air conditioning, space heating, energy recovery from flue gas streams). When exchanging heat with air, the main thermal resistance is located on the air-side. Over the course of the past decades, extensive research has been done on enhancing the performance of liquid-to-gas heat exchangers by modifying the design, as is clear from the vast number of papers published on this topic. And still today, this topic continues to draw a lot of attention. Bergles [2] summarized the idea of evolution in heat exchanger technology by defining 'generations'. Each generation adds a heat transfer enhancement mechanism to the previous one. For this study, generation I consists of tubular heat exchangers, thus having only primary surface. In generation II exterior surface area (fins) are added, resulting in the commonly used fin-and-tube heat exchangers. By adding fins, an extra interesting flow phenomenon occurs: the horseshoe vortex. This is a set of longitudinal vortices which wraps itself around the fin-and-tube junction and flows over the fin surface. These vortices result in a locally thinning of the boundary layer, and thus enhance the local heat transfer rate. This naturally occurring enhancement has been studied in detail in the past. Romero-Méndez et al. [3] used flow visualizations to show that a large reduction of the fin spacing can result in excessive laminarisation of the flow. Any turbulent or vortical motion such as the horseshoe vortex is then quickly dissipated by mechanical blockage and skin friction. Similar findings were reported by Mon and Gross [4]. So there exists a law of diminishing returns for adding fin surface area. Below a certain fin pitch (spacing between two fins) the increase in surface area will in fact reduce local heat transfer coefficients, causing a need for even more surface area. Of course adding more fin surface area will also result in a large increase in pressure drop and material costs. To further enhance the heat transfer performance (i.e. the ability to transfer more heat in a given volume) of fin-and-tube heat exchangers, the convective heat transfer coefficient $h_{\text {ext }}$ has to be increased. This can only be done through flow manipulation, as the heat transfer resistance is the result of the surface temperature distribution, which is closely coupled with the velocity field through the thermal boundary layer. This is done in generation III heat exchangers, which are commonly used today.

\section{Heat transfer enhancement through flow manipulation}

There are two methods of flow manipulation: alterations to the main flow and the introduction or exploitation of secondary flows. In main-flow enhancement, the gross characteristics of the flow are manipulated through geometric changes or pressure variations. In secondary flow enhancement, local flow structures are deliberately introduced. In some cases, it may be difficult to distinguish between main-flow and secondary flow methods because both methods can be present (compound designs).

\subsection{Main flow manipulation}

Today, corrugated or highly interrupted surfaces are widely used in compact heat exchangers. These fins mainly exploit two flow manipulation mechanisms to provide a performance improvement compared to continuous plain fins: (1) these surfaces restart the thermal boundary layer, resulting in a lower average thermal boundary layer thickness and thus a higher average convective heat transfer coefficient; and (2) 
above a critical Reynolds number, these surfaces can cause vortex shedding and the resulting unsteady flow and mixing result in an increased heat transfer.

Commonly used corrugated fin types are the sinusoidal and herringbone wavy fin. Mixing in these fins is accomplished through shear-layer instabilities and the formation of spanwise vortices. Metwally and Manglik [11] studied the impact of the corrugation depth and the Reynolds number on the thermohydraulics of sinusoidal wavy fins. For very low Re or very low corrugation aspect ratio $\gamma$ (ratio of the amplitude to the wavelength) the surface geometry has no effect on the flow and the fluid moves undisturbed through the channel without any recirculation. Increasing $\gamma$ or Re gives rise to fluid recirculation or swirl flows in the troughs. The intensity and flow area coverage of this counter rotating lateral vortex grow with $\gamma$. This change in flow behaviour affects the pressure drop and heat transfer. At low Re the flow behaves as channel flow with a constant $\mathrm{Nu}$ and $f \cdot \mathrm{Re}=\mathrm{cst}$. At higher Re the data diverges away from these regimes as the friction factor and $\mathrm{Nu}$ number increase. By considering the wall shear stress and heat flux distributions Metwally and Manglik [11] argued that the flow can be categorized into two distinct regimes: (1) an undisturbed streamline flow regime and (2) a steady swirl flow regime that is characterized by self-sustained transverse vortices in the troughs. Rush et al. [12] visualized these vortices and the resulting macroscopic mixing using dye injection in scaled up models.

Two widely used interrupted fin designs are the offset strip or slit fin and the louvered fin. But next to these two fin types a large range of different geometries are used, e.g. T’ Joen et al. [13] studied an adapted inclined louvered fin used in a commercially available air conditioning unit. Louvered fins are very popular in automotive applications as combined with flat tubes they result in very compact heat exchangers. In both fin types the fin surface is divided into small sections called 'louvers'. In the slit fin these louvers are parallel to the incoming flow, while in the louvered fin they are set at an angle to the flow (the louver angle). Both the louvered fin and slit fin types have been studied extensively through experiments and numerical simulations. Manglik et al. [14] presented an overview of previous correlations for the slit fin geometry and a new correlation. Based on a large database of experimental data, thermo-hydraulic correlations were derived for the slit fin (Wang et al. [15]) and for the louvered fin (Wang et al. [16]). Next to these full scale heat exchanger tests, some authors have focused on the flow behaviour within these interrupted fin designs, in order to better grasp the physics of the heat transfer enhancement. To this end they have studied only the interrupted section (mostly in a scaled version), neglecting the effects of the tube and the landing area. In both fin types self-sustained unsteadiness is present from a certain Reynolds number which improves mixing and thus the heat transfer rate. This was studied in detail by DeJong and Jacobi [17] for slit fins and DeJong and Jacobi [18] for louvered fins through flow visualisation and mass transfer measurements. Tafti and Zhang [19] performed a detailed numerical study of the transition from steady laminar to unsteady flow in standard louvered fins. They reported that the transition is due to the accumulation of flow perturbations and that it behaves in a gradual manner, starting from a $\operatorname{Re}_{\mathrm{Lp}}$ as low as 300 - 400 with initial wake instabilities and at higher Reynolds numbers $(600-700)$ interior instabilities 
become apparent. Cui and Tafti [20] studied the impact of the actual three dimensional geometry of the landing in louvered fins on the thermo-hydraulics. Strong three-dimensionality was found in the flow structure in the region where the angled louver transitions to a flat landing adjoining the tube surface, whereas the flow on the angled louver far from the tube surface is nominally two-dimensional. Due to the small spatial extent of the transition region, its overall impact on louver heat transfer is limited, justifying two-dimensional studies.

Compared to slit fins, an additional flow characteristic arises in louvered fins: at high Reynolds numbers the flow is deflected, lengthening the flow path. This flow pattern is called 'louver oriented flow'. The fluid particles travel a larger distance through the heat exchanger, resulting in more heat exchange, but at the same time the flow experiences a strong increase in frictional drag. At low Reynolds numbers, thick boundary layers block the passage between the louvers, forcing the flow to pass between the different fins. This is referred to as 'duct oriented flow'. DeJong and Jacobi [18] studied this flow behaviour in detail and reported in agreement with other authors that the transition between the two flow profiles occurs rather sudden around $\mathrm{Re}_{\mathrm{Lp}}=200$. The change in flow behaviour is due to the flow following the path of least resistance - the path corresponding to the lowest overall pressure drop. The degree of flow deflection is usually quantified using the concept 'flow efficiency' $\eta$. This is the ratio of the mean flow angle $\alpha$ to louver angle $\theta$, Eq. (1). Achaichia and Cowell [21] found a strong link between the heat transfer rate of flat tube louvered fin heat exchangers and the mean flow behaviour, relating the Stanton number to $\eta$ (Eq. (2)).

$$
\begin{gathered}
\eta=\frac{\alpha}{\vartheta} \\
S t=1.18 \cdot \eta \cdot \operatorname{Re}_{L p}^{-0.58}
\end{gathered}
$$

Using a large database of validated two dimensional numerical CFD-simulations, Zhang and Tafti [22] determined a correlation for the flow efficiency of louvered fins. Results show that $\eta$ is strongly dependent on geometrical parameters, especially at low Reynolds numbers. Flow efficiency increases with Re and louver angle, while decreasing with fin pitch and thickness ratio. Local heat transfer coefficients in louvered fins presented by Lyman et al. [23] showed the impact of the local thermal field surrounding a louver. If a louver is surrounded by the hot thermal wake of an upstream louver, it will have a lower heat transfer coefficient. It is therefore important, as noted by Tanaka et al. [24] to ensure a good positioning of the different louvers to improve the heat transfer rate of a given fin design. This concept is known as 'thermal wake management'.

\subsection{Secondary flows: longitudinal vortices}

The horseshoe vortex is an example of a secondary flow which enhances the heat transfer rate. It occurs naturally around any protrusion which extends out of the boundary layer. Sedney [5] presented a thorough review on the effects of small protuberances on boundary layer flows. For both laminar and turbulent boundary layers, the effects of a three-dimensional surface bump are qualitatively similar. A system of 
vortices forms near the disturbance, bending around it to be carried downstream in a horseshoe pattern. This secondary flow will appear regardless of the shape of the protuberance; but the location and the height of the protuberance are important. The disturbance height must be comparable to the local displacement boundary layer thickness. Longitudinal vortices caused by surface protrusions were found to persist for more than 100 protrusion heights downstream. Protrusions which are designed to generate strong vortices are called 'vortex generators'. A commonly used geometry is the delta winglet. A review on the heat transfer enhancement using longitudinal vortices was presented by Jacobi and Shah [6]. By positioning a row of vortex generators on the fin surface in between the tubes ('common flow-up') Joardar et al. [7] reported a 16.5\% - 44\% increase in the heat transfer coefficient for Re between 220 and $960(0.7 \mathrm{~m} / \mathrm{s}<\mathrm{V}<$ $1.8 \mathrm{~m} / \mathrm{s})$. The vortex generators result in an accelerated flow due to the nozzle-like passage near the tube walls which can also help to reduce the size of the tube wake, as shown by O’Brien et al. [8]. As a final evolution of generation III heat exchangers, some designs incorporate both main flow and secondary flow enhancement, so-called compound designs. Only a few cases have been presented so far in open literature. Ge et al. [9] investigated the impact of multiple vortex generators on slit fin arrays using PIV and naphthalene sublimation. They reported a heat transfer enhancement of $32 \%$ at $\mathrm{Re}_{\mathrm{Lp}}=1000$ for two rows of delta wings, one array at the inlet and one at half the flow length. Joardar et al. [10] studied the performance of a louvered fin heat exchanger with a row of vortex generators mounted upstream of the first tube row. The aim was to generate strong coherent vortices within the fin channels. They reported an average increase of $21 \%$ and $23.4 \%$ in the heat transfer coefficients respectively for dry and wet cases compared to the base case (only louvered fins). The pressure drop increased by only $6.6 \%$ on average. The inlet velocity varied between $1.5 \mathrm{~m} / \mathrm{s}$ and $4 \mathrm{~m} / \mathrm{s}$. These results clearly show the promise of this new technique to further augment the heat transfer of existing fin designs.

The literature survey presented above summarizes the ideas behind the evolution of modern compact finand-tube heat exchangers up to today. A similar review up to 1998 was provided by Jacobi and Shah [25]. The results show the strong interaction between flow behaviour and thermo-hydraulics for the different surfaces. So by understanding the flow physics which (can) occur, it is possible to seek out ways of improving a current design through foresight, rather than by trial-and-error. Fin designs should focus on reducing the average thermal boundary layer thickness, without incurring excessive frictional drag. With this in mind, this research study was performed, in which a hybrid fin design, the inclined louvered fin, was studied.

\section{Inclined louvered fins}

The inclined louvered fin is a hybrid design of louvered and offset strip fins, as described by Shah et al. [26]. The plates are aligned with the main flow (Fig. 1) but are set out in a staggered layout, forming a stair like deflecting channel. In Fig. 1 the main geometric parameters are indicated: the fin pitch $\mathrm{F}_{\mathrm{p}}$, the louver pitch $L_{p}$, the fin thickness $t$, the fin angle $\varphi$ and the number of louvers. Just as in louvered fins there are 
distinct inlet-, turnaround- and exit-louvers. The design shown in Fig. 1 represents the interrupted section of the fin surface between the tubes. This interrupted section needs to be connected to the tubes to form the heat exchanger, which is usually done using a transition to a flat fin surface (landing). Cui and Tafti [20] showed that the impact of the landing and transition area on the flow is focused close to the tube wall, so the interrupted element can be studied separately. To manufacture these fins a complex series of machining processes such as stamping, rolling... will be required, but considering current multi-louvered and corrugated designs in production, there appear to be no significant barriers to the production of the proposed design.

Tanaka et al. [24] previously studied inclined louvered fins and considered another parameter: the louver angle, which is set at $0^{\circ}$ in the current study. They used a two-dimensional analytical model to study the flow behaviour. The primary aims were to ensure that the air flow passed straight through the fin array, avoiding deflection to reduce the air-side pressure drop, and to enhance the heat transfer coefficient by positioning each louver outside of the boundary layer of an upstream louver. The flow in several scaled up models was visualized: for high fin angles $\left(35^{\circ}\right)$ deflection and strong wakes were noted, while for small angles $\left(10^{\circ}\right)$ a strong developing boundary layer was found. An intermediate fin angle $\left(20^{\circ}\right)$ resulted in an almost straight flow with little separation or deflection. After the scaled model tests, a single heat exchanger was built, tested and compared to a louvered fin. In the considered velocity range $(1-5 \mathrm{~m} / \mathrm{s})$ the average heat transfer coefficient of the inclined louvered fin core was $16 \%$ higher and the pressure drop 21$27 \%$ lower. This example clearly indicates the potential of this fin type. No other experimental data have been reported so far.

If the geometry of the inclined louvered fin (Fig. 1) is considered, a priori it is clear that similar phenomena as in louvered fins will occur. At low Re the thick boundary layers will block the passages between the louvers, forcing the flow to wind up and down; while at high Re, the flow passages open up and the flow becomes more slit fin like, aligned with the louvers. This expected change in flow behaviour is the main reason why the inclined louvered fin geometry was selected for this study. As the flow deflection occurs at low Reynolds numbers, this fin type could present an interesting alternative to louvered fins. Just as in other interrupted fin designs it is expected that from a certain Re unsteady flow will be present in the fin array, which enhances local heat transfer. In order to study the interaction between the flow behaviour and the thermo-hydraulics, scaled up models of the fin will be used and a combination of experimental (flow visualization and local heat transfer coefficient measurements) and numerical techniques. As can be seen in Fig. 1, there are five potentially important geometric parameters: fin pitch, louver pitch, fin angle, number of louvers and fin thickness. Using a numerical screening study (T'Joen et al. [27]) it was shown that the fin pitch and fin angle had the strongest impact on the thermo-hydraulic behaviour, so these parameters are studied in more detail. 


\section{Flow behaviour}

\subsection{Experimental set-up and procedure}

The flow field was studied by visualizing the flow in a water tunnel through dye injection. In the closed loop water tunnel (located at the University of Illinois), the water is circulated using a frequency controlled pump through a plenum, followed by a contraction section, the test section and then finally a reservoir. To ensure a uniform flow at the inlet of the test section, two honeycomb flow straighteners are used in the contraction. The test section has a length of $0.4 \mathrm{~m}$, a width of $0.3 \mathrm{~m}$, a height of $0.3 \mathrm{~m}$ and is made of transparent Plexiglass. A mirror mounted at $45^{\circ}$ underneath the test section allows for convenient recording of the flow images using a digital camera. The water temperature was measured using a K-type thermocouple. In order to determine the water velocity, the time required for a dye marker to travel a given distance $(0.25 \mathrm{~m})$ was measured using a stop watch. To visualise the flow, dye is injected at a specific point just upstream of the test section, highlighting a single streakline. Scaled models (20:1) of the inclined louvered fin were placed in the test section. Five configurations with varying geometric parameters were studied (Configurations 1-5 in Table $1, \mathrm{~L}_{\mathrm{p}}=0.02 \mathrm{~m}, \mathrm{t}=0.002 \mathrm{~m}$ ). The scaling factor was selected in order to obtain sufficient spatial resolution, while maintaining a sufficiently large number of fin rows to ensure periodic flow behaviour. DeJong and Jacobi [28] showed that wall effects can result in a strong distortion of the measured mean flow angle in louvered fins and they presented a calculation method to determine the minimal number of fin rows required. This method was used to guide the scaling factor of the fin array. The number of fin rows in the scaled models ranged between 8 and 14 depending on the fin pitch. More details on the measurement setup and procedure and the uncertainty analysis can be found in T'Joen et al. [29].

\subsection{Mean flow behaviour: fin angle alignment factor}

In order to quantify the flow behaviour in the inclined louvered fins, the 'fin angle alignment factor' $\zeta$ (defined in Eq. (3) as the ratio of the mean flow angle $\alpha$ to the fin angle $\varphi$ ) is introduced. Note that the definition is identical to that of the 'flow efficiency' $\eta$ for standard louvered fins. For louvered fins, a high value of $\eta$ corresponds to a flow almost parallel to the louvers, resulting in large heat transfer coefficients due to thin boundary layers. Thus high values of $\eta$ are good from a heat transfer perspective, resulting in the term flow 'efficiency' for $\eta$. For the inclined louvered fin array, high values of $\zeta$ indicate that the flow along the louver surface is dramatically reduced, and the local heat transfer coefficient can be expected to be reduced. This would make the term 'flow efficiency' misleading for inclined louvered fins, thus an alternate term is used, the 'fin angle alignment factor'.

$$
\varsigma=\frac{\alpha}{\varphi} \approx \frac{\tan \alpha}{\tan \varphi}
$$

For each of the five configurations flow images were recorded at various Reynolds numbers. At each $\operatorname{Re}_{\mathrm{Lp}}$ the dye injection point was varied over the channel width. This resulted in a series of images of various streaklines which, once combined, provide an overview of the total flow field at that Reynolds number. For each of the flow images, $\zeta$ can be determined graphically based on the approximation in Eq. (3) and by 
using a graphical software package. The mean flow angle was determined between the point where the streakline enters the fin array and the midpoint of the turnaround louver. For each configuration a series of graphs can then be made, one per Reynolds number, showing the local values of $\zeta$ set out against the injection location. This value represents the position of the injector relative to the fin passage and is scaled with the fin pitch, so it ranges from 0 (top of the fin passage) to 1 (bottom of the fin passage). An example is shown in Fig. 2 (filled symbols). The error bars are indicated. There is clearly a strong variation depending on the injection location, which is due to the turnaround louver: a streamline which just passes above or below the turnaround louver will have a higher or lower $\zeta$ compared to a streamline which passes at a distance of the turnaround louver. Similar findings were reported by DeJong and Jacobi [18], who found a maximum flow efficiency value for a streamline in the center of the fin passage, and a minimum for a streamline touching the inlet louver. In their data reduction the average of this minimum and maximum was used. It is clear from Fig. 2 that using the average of the maximum and minimum value of the experimental data points would not represent a fair average of $\zeta$. So, to determine a correct average value the gaps between the data points should be filled with additional data. This was done by using numerical simulations.

\subsection{Numerical set-up and procedure}

The flow through the five different configurations was simulated using Fluent ${ }^{\odot}$ (double precision segregated solver). Two dimensional cases were studied using unstructured quadrilateral meshes generated by Gambit ${ }^{\odot}$. A single louver row was simulated with an entry region (two fin pitches) and exit region (five fin pitches). The height of the computational domain is set to one fin pitch with periodic boundary conditions on the top and bottom of the domain, representing the periodic unit of an infinite stack of fins. A uniform inlet velocity in the $\mathrm{x}$-direction (parallel to the louvers) was imposed. At the outlet the static pressure was set to $0 \mathrm{~Pa}$ (pressure outlet boundary condition). Convergence criteria were set to $10^{-8}$ for continuity and velocity components. Second order discretization was used in combination with the SIMPLE algorithm for pressure-velocity coupling. Substance properties were set to a constant value. The average pressure drop and local velocity components were monitored during the iterations to determine if the simulations had converged. If no steady state convergence was obtained, an unsteady simulation was performed till the different monitored signals (pressure drop, local velocity in the wake of the louvers and mean outlet velocity) showed a clear periodic behavior. For those cases data was averaged out over 10 - 15 periods of the signal. A grid independence study was performed. More details on the numerical setup and benchmarking can be found in T'Joen et al. [30].

In order to determine $\zeta$ from the CFD simulations, the same procedure was used as for the water tunnel experiments. The streamlines were visualized using the 'path lines' option in Fluent ${ }^{\mathbb{Q}}$ and the resulting image was exported to the graphical software. The same data reduction was performed as for the experimental flow images which resulted in a set of numerical data points, spread evenly out over the fin 
spacing. The results are shown in Fig. 2 (open symbols). As can be seen, the agreement between the simulations and the experimental data is very good (the difference is for most data points smaller than 0.05). This was the case for all considered Reynolds numbers. As this method of determining $\zeta$ is tedious, an alternative method was used as well. For each louver (excluding the inlet, turnaround and exit louver) an average flow angle $\alpha_{\mathrm{L}}$ over the fin passage was determined based on the ratio of the average values of the $\mathrm{x}$ - and $\mathrm{y}$ - component of the velocity. These averages were determined over a cut perpendicular to the louver spanning the fin passage, intersecting the louver at the center. The $\alpha_{\mathrm{L}}$ values are then averaged out over all the louvers and divided by the fin angle to determine the fin angle alignment factors, labeled $\zeta_{\mathrm{T}}$. Downstream of the turnaround louver, the average flow angle is divided by the negative fin angle as the flow now points down instead of up. This is described in more detail in T'Joen et al. [30]. This definition of $\zeta_{\mathrm{T}}$ makes sense, recalling the original goal of this parameter, which is to quantifiably describe the mean flow behaviour in the louver passages. Comparing the graphically measured data with the $\zeta_{\mathrm{T}}$ values revealed strong differences. By comparing graphically measured $\zeta$ values based on different measurement locations (varying the points in between which the flow angle is measured) revealed that the graphical measurement can be biased due to the occurrence of recirculation zones. Through extensive comparison it was shown (T'Joen et al. [30]) that $\zeta_{\mathrm{T}}$ is the correct measure for the mean flow behaviour surrounding the louvers. Using the validated numerical method described above, 8 additional configurations were studied (configurations 6-13 in Table 1). The results for configuration 1-5 are shown in Fig. 3. From the results it is clear that flow deflection occurs at low Reynolds numbers, but that it is less pronounced than in louvered fins: Zhang and Tafti [22] and DeJong and Jacobi [18] reported $\eta$ values between 0.5 and 0.95 in the same $\mathrm{Re}_{\mathrm{Lp}}$ range. The data in Figure 3 also shows that if the fin angle is not too small (configurations $3-4-5$ ), the flow deflection is nearly constant in the considered Reynolds range (50-500, consistent with air conditioning applications). This is in contrast to louvered fins, which show a sharp reduction in $\eta$ (and thus become less effective) once $\mathrm{Re}_{\mathrm{Lp}}$ drops below 200. The flow deflection also shows an opposite trend in inclined louvered fins, increasing as the Reynolds number decreases. The data shows that decreasing the step size (smaller fin angle), and increasing the fin pitch ratio (ratio of $\mathrm{F}_{\mathrm{p}}$ to $\mathrm{L}_{\mathrm{p}}$ ) results in stronger flow deflection. This is due to a combination of boundary layer growth on the louvers (smaller passages are more easily blocked) and the flow following the path of least resistance (a wider channel in between the fins than in between the louvers favors flow deflection). These results show how the flow in the inclined louvered fin array is driven by the growth of boundary layers on the louvers.

\subsection{Transition to unsteady flow}

During the flow visualization the transition from steady laminar flow to unsteady flow was also studied. It was found that the initial instability appears in the wake of the exit louver. As the Reynolds number increases the flow instabilities move upstream into the fin array. The onset of unsteady flow occurred at low Reynolds numbers (200-300). Figure 4 shows the Reynolds numbers at which the first unsteady flow behaviour was detected at the inlet-, turnaround- and exit-louver for configurations 1-5. It can be seen that 
the fin pitch and fin angle both impact the transition to unsteady flow: e.g. decreasing the fin pitch postponed transition to much higher Reynolds numbers for a small fin angle. A detailed description of the transition for each configuration is presented in T'Joen et al. [29]. As a reference numerical data by Tafti and Zhang [19] for two louvered fin cases were added to Fig. 4. As can be seen, the transition in the inclined louvered fin configurations occurs at much lower Reynolds numbers. Tafti and Zhang [19] showed that both in louvered and slit fins the transition is due to the accumulation of perturbations in the flow and that it behaves in a gradual manner. The louvers act as individual roughness elements perturbing the flow and the cumulative effect of these perturbations causes the flow to develop instabilities. The transition in the inclined louvered fins clearly is different, as it moves much faster upstream into the fin array (Fig. 4). It was shown (T'Joen et al. [29]) that the transition is geometrically triggered by the large recirculation zones which are present on the inclined parts of the inlet and turnaround louver. At low $\operatorname{Re}_{\mathrm{Lp}}$ steady recirculation bubbles form, but as the velocity increases the shear layers trigger unsteady flow and vortex shedding becomes apparent. The previously studied louvered fin designs all had a much smaller inclined section on the turnaround louver, which is why this phenomenon has not been reported so far. The experimental findings agree well with the numerical simulations both for the predicted Reynolds number at which unsteady flow first appears as well for the way the unsteadiness moves rapidly upstream into the fin array as the Reynolds number is increased. To quantify this transition the local flow velocity was recorded in the wake of each louver $0.5 \mathrm{~L}_{\mathrm{p}}$ away from the trailing edge. Once periodic conditions were achieved, the ratio of the amplitude of the velocity variation to the mean value was set out for each louver. The results for configuration 4 at different Reynolds numbers are shown in Fig. 5. The initial unsteadiness was found at $\mathrm{Re}_{\mathrm{Lp}}=295$ in the wake of the exit louver. At $\mathrm{Re}_{\mathrm{Lp}}=316$ the entire downstream half of the fin array experiences periodic unsteady flow (Fig. 5), but in the upstream half of the fin laminar flow is present. Increasing the Reynolds number results in stronger oscillations and at $\operatorname{Re}_{\mathrm{Lp}}=550$ the upstream of the fin array also shows strong periodic oscillations apart from the wake of the inlet louver. $\mathrm{At}_{\mathrm{Lp}}=800$ the flow behaviour in the entire fin array has become very chaotic with strong oscillations (Fig. 5: ratio $\sim 1$ ). Similar agreement was found for the other cases, see T'Joen [31].

\section{Thermo-hydraulics}

\subsection{Experimental set-up and procedure}

To determine the thermo-hydraulic characteristics of the inclined louvered fin, a series of wind tunnel experiments was performed on the scaled models. The test rig is an open wind tunnel, as shown in Fig. 6, consisting of a large centrifugal fan (3) driven by a frequency controlled drive (2+1). The fan blows the air through a large settling chamber filled with a honeycomb (4) (section: $1.10 \mathrm{~m}$ x $1.10 \mathrm{~m}$ ). The air then passes through a contraction section (5) with sinusoidal shaped walls to generate a uniform flow at the inlet of the settling channel (6) (3m long, section: $0.4 \mathrm{~m} \times 0.2 \mathrm{~m}$ ). The area contraction ratio is 15 , which is larger than the recommended 6 - 9 by Mehta and Bradshaw [32] for small size wind tunnels. The air then flows through the test section (7) and a final channel section (8) of $1 \mathrm{~m}$. This section was added to prevent 
any upstream influence of the flow contraction as it enters the downstream tube. The air mass flow rate is measured using a calibrated orifice plate (9) (designed and installed according to the standard ISO 5167) set in the downstream tube (I.D. = $18.29 \mathrm{~mm}$ ). The test section and downstream channel were insulated using $5 \mathrm{~cm}$ thick polystyrene plates to reduce any heat losses to the ambient. The test section contains the scaled fin model (20:1). The same scaling factor as for the water tunnel experiments was used based on a similar compromise between spatial resolution (measuring the local wall temperature using embedded thermocouples) and the need for having sufficient fin passages to prevent any wall effects. As the wind tunnel is higher than the water tunnel the fin pitch ratio could be increased up to 2 while maintaining periodic flow conditions. Wall pressure taps were mounted at the side of the test section to measure the pressure drop with a piezoresistive sensor (LP1000, GE Druck, range: 25 Pa, accuracy: 0.125 Pa). As a benchmark experiment the test section was filled with narrow (8 $\mathrm{mm}$ high) $0.26 \mathrm{~m}$ long rectangular channels. The pressure drop was recorded and the isothermal friction factor $\mathrm{f}$ (Kays and London [33], Eq. (4)) was determined. All pressure drop measurements were done without heating. The results showed a very good agreement with laminar developing flow theory as presented by (Shah and London [34]).

$$
f=\frac{2 \cdot \rho \cdot \Delta P \cdot A_{c}}{G_{c}^{2} \cdot A_{e x t}}
$$

The walls of the test section were made out of $3 \mathrm{~mm}$ thick balsa wood. This material was selected as it allows for easy manual cutting of the complex fin shapes without any machining. The louvers are made out of print board material ( $2 \mathrm{~mm}$ thick) coated with a thin copper layer on both sides. To provide heating, a current was sent through the copper layer resulting in a uniform heat flux boundary condition. The current is controlled using an external power supply and measured using a current meter. The maximum current sent through the louvers is $10 \mathrm{~A}$ (maximum heat flux $=187.5 \mathrm{~W} / \mathrm{m}^{2}$ ). The different louvers are connected in series to each other electrically using wires, ensuring the same heat flux is dissipated throughout the fin array. The top and bottom of each louver are connected using soldered copper contacts. Only five louver rows were heated: those surrounding the center row where the temperature measurements are done. It is important to heat the adjacent fin rows in order to accurately capture any thermal wake effects, as shown by Lyman et al. [23]. By measuring the local air velocity (using a pitot tube) and temperature in the wake of the fin array the heat balance was verified to be within $5 \%$.

To determine the local heat transfer coefficient, the surface temperature $\mathrm{T}_{\text {surf }}$ must be determined. This was done using a measurement louver made from balsa wood in which nineteen K-type thermocouples (0.5 mm thickness) were embedded. The junctions were aligned in the center of the louver along the flow direction in order to measure the surface temperature change along the flow path. The balsa wood was covered with a layer of paper and then a thin copper foil was glued on top. The thermocouple junctions just pierced the paper. To ensure a good contact between the copper foil and the junctions, these were covered with thermally conductive paste before the foil was glued on. Current was sent through the measurement louver to provide heating, but as the resistance of the copper foil differed a bit from the resistance of the copper 
film on the other louvers a separate control circuit was used to ensure all louvers dissipated the same heat flux. The thermocouples were calibrated individually using a reference thermometer, which resulted in an uncertainty of $0.1^{\circ} \mathrm{C}$ on the measured surface temperatures. The heat flux was set depending on the air velocity and this by monitoring the surface - air temperature difference to minimize the impact of natural convection. The temperature difference was limited to $4{ }^{\circ} \mathrm{C}$ at the lowest $\operatorname{Re}_{\mathrm{Lp}}$ which resulted in $\mathrm{Ri} \sim 0.16$.

The measurements were taken at steady state conditions. To determine that transient effects (start up or changing of incoming air mass flow rate) had ceased the surface temperature variation was monitored. Once steady state was achieved the measurements were started and the data-acquisition system recorded the surface temperature and inlet temperature values for a period of 2 minutes every 2 seconds. Once this was done, the measurement louver was placed at the next position, and as soon as steady state was again achieved, the temperatures on this position were recorded. This was done for all louver positions. The average values of the signals were used in the data reduction.

\subsection{Data reduction}

To determine the local heat transfer coefficient, Eq. (5), three variables are needed: the surface heat flux q, the surface temperature $T_{\text {surf }}$ and a reference temperature $T_{\text {ref. }}$ In the experiments the heat flux is imposed and thus known (by measuring the current and the resistance of the louvers), and the local surface temperatures are measured. As a reference temperature, the inlet air temperature was used to allow for an easy comparison with other researchers. Alternative reference temperatures include the bulk mean temperature (which can result in biased values due to thermal wake effects, as shown by Zhang and Tafti [35] and Lyman et al. [23]) or the adiabatic mean temperature as introduced by Moffat [36]. The latter represents the actual driving potential for heat transfer and enables the effects of the flow field and thermal field to be separated. However, hardly any data is available on other fin types providing heat transfer coefficient values based on this temperature and using this temperature increased the time required for the measurements significantly.

$$
h=\frac{q \pm q_{\text {cond }}}{T_{\text {surf }}-T_{\text {ref }}}
$$

The core of the measurement louver was constructed of balsa wood. During the measurements a temperature difference was recorded between the top and bottom of the louver, which will result in a heat flux $\mathrm{q}_{\text {cond }}$ flowing through the louver. This must be taken into account when calculating the heat transfer coefficient. This conductive flux can be calculated based on the thermal conductivity of the balsa wood ( $0.0453 \mathrm{~W} / \mathrm{mK}$, measured using guarded hot plate - ISO 8302), the louver thickness and the measured temperature difference (Eq. (6)). The impact of the copper foil, paper and thermal conductive paste were negligible. Even though $\mathrm{k}_{\mathrm{balsa}}$ is very low, because the louver is thin it still results in a significant conductive heat flux through the louver, even for small temperature differences between the top and bottom. By assuming one dimensional conduction through the louver (perpendicular to the surface), the 
conductive heat flux can be computed based on the measured local surface temperatures. As the stream wise temperature differences were small, this is considered a reasonable approximation. By adding or subtracting $\mathrm{q}_{\mathrm{cond}}$ to/from the imposed heat flux q, the actual heat flux is known (Eq. (5)).

$$
q_{\text {cond }}=\frac{k_{\text {balsa }}}{t_{\text {balsa }}} \cdot\left(T_{\text {top }}-T_{\text {bottom }}\right)
$$

To determine the average heat transfer coefficient, the local values were first averaged out for a louver and then the average was determined for the whole fin array. Due to the smaller size, it was not possible to determine the local h values with sufficient accuracy on the inlet, turnaround and exit-louver. So no average values will be presented for these louvers. The local temperature measurements did reveal the presence of the recirculation zone on top and underneath the turnaround louver: large temperature difference between the top and bottom and a sharp gradient from leading to trailing edge. In order to be able to indicate the quality of the measurements a thorough error analysis was made using the procedures found in Moffat [37]. The uncertainty on the viscosity and thermal conductivity of the air in the calculations was $2 \%$ (Kadoya et al. [38]). The air density was determined through the ideal gas law and the uncertainty was determined through error propagation based on the environmental temperature and pressure measurement. Based on the uncertainty in the resistance (1.25\%) and current measurements (0.05 A) an average uncertainty of 3.6\% was found for the imposed heat flux q. The uncertainty on qcond was determined based on the uncertainty of the temperature difference and of the thermal conductivity of the balsa wood (1.5\%). This resulted in an uncertainty ranging from $5 \%$ to $16 \%$ on the local h values.

\subsection{Numerical setup}

To gain more quantitative insight into the local flow behaviour (e.g. the thermal boundary layers) a number of two and three dimensional numerical simulations were performed including heat transfer. Even though the flow visualization provides a great tool to study the mean flow behaviour, and specific local phenomena such as separation and unsteadiness, it was found that more detailed information was needed to link the measured data to the occurring flow physics. The same simulation settings as described above (4.3) were used. In addition, a uniform heat flux boundary condition was imposed on the louvers, and the required residual for convergence of the energy equation was set to 1e-6. Second order discretization was used for the energy equation. The air substance properties remained constant apart from the density (set to 'incompressible ideal gas') and the dynamic viscosity (set to the 'Sutherland law') which were allowed to vary as a function of temperature. For the three dimensional cases the two dimensional models of the fin were extruded. The width equaled half the test section width $(0.1 \mathrm{~m})$. A symmetry boundary condition was imposed on one side wall and a no-slip condition on the other (= channel wall).

A comparison between the numerical (both two and three dimensional simulations) and experimental friction and Colburn factor data is shown for configuration 2 in Fig. 7A. As can be seen, the numerical results agree well with the experimental data for both heat transfer and pressure drop. The difference 
between the 2D and 3D CFD values of the Colburn factor is very small. This is due to the flow acting nominally two dimensional. This is illustrated in Fig. 7B in which the louver averaged span wise heat transfer coefficient is set out as a function of the distance to the wall $(\mathrm{z}$, in $\mathrm{m})$. The coordinate $\mathrm{z}=0$ corresponds to the channel wall, while $\mathrm{z}=0.1 \mathrm{~m}$ is the center of the channel. The values represent the average heat transfer coefficient over a louver length at different $\mathrm{z}$ coordinates. Figure 7B shows that the impact of the boundary layer on the channel wall is confined to a small section of the channel, and even there the drop off is not that sharp, as the values only reduce by about $20 \%$ at the point closest to the wall ( $\mathrm{z}$ $=0.005 \mathrm{~m}$ ). Figure 7B shows data for the lowest considered value of $\mathrm{Re}_{\mathrm{Lp}}$, which had the strongest impact of the boundary layer. This two dimensional flow behaviour had also been noted during the experiments, as temperature measurements using the measurement louver at different locations in the channel section $(0.25$ of the width, in the center and at 0.75 of the width) revealed no difference within measurement uncertainties. Figure 7A shows a slight difference between the 2D and 3D values for the friction factor. This is due to the added flow friction by the channel wall. As can be seen the experimental $f$ values agree well with both cases, but the difference with the 3D cases is slightly smaller.

\subsection{Results and discussion}

An example of a heat transfer coefficient measurement is shown in Fig. 8 for configuration $\underline{1}$ (Table 1) $\operatorname{Re}_{\mathrm{Lp}}$ $=185$. As a dimensionless coordinate $\mathrm{x}$-coordinate $\mathrm{X}^{*}$ (Eq. (7)) is introduced. Positive values represent the top side of the louver, negative the bottom side. The definition shows that 0 represents the leading edge of the louvers, while 1corresponds to the trailing edge. The heat transfer coefficients on the top side of the upstream louvers show the typical boundary layer behaviour: a sharp drop off starting from the leading edge. The bottom side however of these louvers shows the opposite trend, increasing towards the trailing edge. To explain these trends the local velocity field around L3 is shown in Fig. 9A. On the top side of the louver a boundary layer grows starting from the leading edge. Because the flow is only slightly deflected $(\zeta$ $\sim 0.63$, corresponding to a mean flow angle of about $8^{\circ}$ ) the boundary layer growth is very similar to that of a flat plate parallel to the flow. Near to the trailing edge the boundary layer becomes thinner due to the local flow acceleration as a small portion of the flow is deflected between the louvers. This result in higher heat transfer coefficient values near the trailing edge, a phenomenon also noted by DeJong and Jacobi in slit fins [17]. On the bottom side of the louver the boundary layer starts to grow from the leading edge. Due to the presence of the accelerating flow from in between the louvers the local flow angle to the louver surface is increased, thickening the boundary layer. Further downstream on the bottom the soft impingement of the main flow thins the boundary layer as it approaches the trailing edge, resulting in the 'inversed' heat transfer coefficient profile. In the second half of the fin array (L6 - L10) the trends are reversed as the top edges now show an 'inversed profile', though to a lesser extent. The top edges of the upstream louvers have the highest $\mathrm{h}$ values. As stated above the heat transfer coefficient is determined using the inlet temperature as a reference value. If the flow would be perfectly mixed after every louver using $T_{\text {in }}$ as a reference would result in a steady dropping value of $h$ as the heat flux is fixed. Instead, the 
temperature distribution is very non uniform, exposing the top surfaces of the upstream and the bottom surfaces of the downstream louvers to cooler air, raising their $\mathrm{h}$ values. This is illustrated in Fig. 9B. This example clearly illustrates the coupling between the local flow behaviour and the heat transfer coefficients.

$$
X^{*}= \pm \frac{X-X_{\text {leadingedge }}}{L_{p}}
$$

By averaging out the local heat transfer coefficients per louver, the impact of the change in flow behaviour can be visualised in the fin array at different Reynolds numbers. Figure 10 shows an overview of the numerically determined averaged heat transfer coefficients per louver for different Reynolds numbers for configuration 2. As is shown, at low Reynolds numbers the fin acts as if it was a plate with a growing boundary layer: the averaged convection coefficients gradually decreased downstream. At low Re the flow is nearly aligned with the fin angle (Fig. 3), transporting all heat from the louvers following the shape of the fin array (strong 'intra-fin' interference, Zhang and Tafti [35]). As $\mathrm{Re}_{\mathrm{Lp}}$ increases, the mean flow angle reduces as the fin passages open up. As a result the thermal wakes of the louvers are deflected more away from the fin array, resulting in an increase of the convection coefficients moving downstream in the fin array. Louvers 6 and 7 present the lowest louver averaged heat transfer coefficient values at higher $\mathrm{Re}_{\mathrm{Lp}}$. This is due to thermal wake interference: as the flow angle reduces in value (Fig. 3) the hot wakes of L4, L5 and the TL are directed towards L6 and L7. This results in local reductions of the heat transfer coefficient. This is consistent with the experimental results of Springer and Thole [39] who studied the flow in different louvered fin arrays. They reported that at higher $\mathrm{Re}_{\mathrm{Lp}}$ the hot wakes of the louvers became narrower but persisted over a larger stream wise distance. This explains the sharp drop at L6. As the flow angle reduces the louvers are exposed to cold air from the inlet which results in higher heat transfer coefficient values in the downstream half of the fin array.

Based on the average heat transfer coefficient for all the louvers, the Colburn j-factor can be determined for the fin array (Eq. (8)). An overview of the experimentally and numerically determined (based on two dimensional cases) Colburn j-factor and friction factor is presented in Fig. 11A and 11B respectively for configurations $1-9$. Only these configurations are shown to avoid overcrowding the figure while still highlight the trends in the data-set. The remaining configurations showed the same trends. As shown already in Fig. 7, good agreement is found between the numerical and experimental data. So it was chosen to only list numerically determined values in Fig. 11B, as data points with error bars would overcrowd the graph. Figure 11A shows a combination of experimental data and numerical values due to the long computational times required for the unsteady simulations with heat transfer. Figure 11C shows the corresponding fin angle alignment factor values $\zeta_{\mathrm{T}}$. It is clear from this figure that at a given inlet velocity $\left(\operatorname{Re}_{\mathrm{Lp}}\right)$, the resulting flow behaviour is very dependent on the fin geometry. It can vary from strong flow deflection following the stair like channel to almost slit-fin like flow. Configurations with a small fin angle and high fin pitch ratio are prone to channel flow, while configurations with a high fin angle are prone to 
slit fin like flow. The absolute value of the mean flow angle ranged between $0.4^{\circ}$ (configuration $4, \operatorname{Re}_{\mathrm{Lp}}=$ 550 ) and $15.8^{\circ}$ (configuration $6, \operatorname{Re}_{\mathrm{Lp}}=20$ ).

$$
j=\frac{\bar{h} \cdot L_{p}}{k_{a i r} \cdot \operatorname{Re}_{L p} \cdot \operatorname{Pr}^{1 / 3}}
$$

Examining the friction factor data (Fig. 11B) shows that increasing the fin angle and reducing the fin pitch results in higher friction factors. A smaller fin pitch results in a more compact structure, exposing the flow to more surface friction. It also reduces the $\zeta_{\mathrm{T}}$ values (Fig. 11C), which means the flow is better aligned with the louvers, so the flow experiences more frictional drag. If the $\zeta_{T}$ value is high, the flow follows the channel and the louver surfaces (particularly the frontal one) experience relatively low velocities around it (as shown in Fig 9A), which reduces form and frictional drag. As the $\zeta_{T}$ values reduce, the velocities around the louvers increase and the flow experiences an increasing amount of drag. This also explains the impact of the fin angle as increasing the fin angle reduces the flow deflection. Additionally a larger fin angle also results in a slight increase in the frontal surface area of the inclined parts (IL, TL and EL) which increases the form drag. As a reference value, Fig. 11B contains the friction factor for fully developed laminar channel flow (solid black line). At low $\mathrm{Re}_{\mathrm{Lp}}$, the friction factor of configurations with a small fin angle is almost parallel to this line but higher. The flow does behave as in a channel but due to the extra form drag the friction factor is higher. As $\mathrm{Re}_{\mathrm{Lp}}$ increases, a transition occurs as the friction factors move away from solid line. This corresponds to a drop in $\zeta_{\mathrm{T}}$ : the flow becomes more aligned with the louvers and less channel like. Cases with a high fin angle also result in friction factors which behave nearly parallel to the solid line at low $\mathrm{Re}_{\mathrm{Lp}}$, but at much higher values. For these cases the flow is aligned with the louvers, flowing straight through. The flow experiences the louvers as obstacles (in particular the inclined sections) in the channel which results in high friction factors. From a certain $\operatorname{Re}_{\mathrm{Lp}}$ value the curves gently start to move away from the solid line. This is due to the onset of unsteady flow (Fig. 4-5): the onset of large scale mixing results in a larger pressure drop. This transition is less pronounced than the change due to the drop in $\zeta_{\mathrm{T}}$ values for the cases with a small fin angle. The friction factor of the cases with an intermediate fin angle value show a mixed behaviour as they are already moving away from the solid line at the lowest $\operatorname{Re}_{\mathrm{Lp}}$ considered here (corresponding to their change in flow deflection). Additionally starting from $\operatorname{Re}_{\mathrm{Lp}} \sim 400$ unsteady flow appeared and started to move upstream into the fin array. As a result the flow never behaves channel like.

Comparing the heat transfer data in Fig. 11A to the $\zeta_{\mathrm{T}}$ values shows a similar agreement in trends as for the friction factors. For configurations with a small fin angle an increase of the fin spacing results in a reduction of the Colburn factor. As the fin passage becomes larger, more air flows through the passage without making contact with the fin surface (see e.g. Fig. 9) resulting in a decreased heat transfer rate. As $\mathrm{Re}_{\mathrm{Lp}}$ increases, a transition occurs as the Colburn j-factor curves start to decrease less fast with $\mathrm{Re}_{\mathrm{Lp}}$ compared to at low $\mathrm{Re}_{\mathrm{Lp}}$. This transition corresponds to the change in flow behaviour: as the louver passages open up, the flow becomes better aligned with the louvers resulting in higher heat transfer 
coefficients. For configurations with a larger fin angle an increase of the fin pitch results in a small increase of the Colburn j-factor. This is due to inter-fin interference: as the fin angle increases, the flow alignment decreases, resulting in flow that passes straight through the fin. The hot wakes of the louvers in the upstream half of the fin array will then be transported down towards the downstream louvers, resulting in (strong) reduction of the heat transfer coefficients. By increasing the fin pitch the flow is slightly more deflected resulting in less interference. Also, by increasing the fin pitch the stream wise distance between two louvers is increased, reducing the interference. Springer and Thole [39] showed that at high $\mathrm{Re}_{\mathrm{Lp}}$ the louver wakes become narrower and persist over a greater distance through the fin array. So at high $\mathrm{Re}_{\mathrm{Lp}}$ the interference should increase again, reducing heat transfer coefficients. But as the flow becomes unsteady at these velocities, the flow gets mixed, breaking up the wakes. Similar to the friction factors, the Colburn $\mathrm{j}$ factors of the configurations with an intermediate and high fin angle show a relative increase compared to the trend of the low $\operatorname{Re}_{\mathrm{Lp}}$ data due to the onset of unsteady flow.

These results clearly highlight the link between the mean flow behaviour (deflection and unsteadiness) and the thermo-hydraulics in the inclined louvered fins. This link is due to the boundary layers on the louvers and the onset of unsteady flow. As was shown, the boundary layers on the louvers drive the flow deflection and also govern the local heat transfer which is then averaged out over the array. By aligning the flow to the louvers the boundary layers become thinner and heat transfer increases. At the same time the pressure drop increases as the flow experiences more frictional and form drag. Through a better understanding of the flow physics occurring within fin designs, a further improvement of the performance could be achieved. In particular this study showed how flow behaviour can be manipulated through a choice of geometric parameters. It was shown that by using large inclined sections unsteady flow can be geometrically triggered at low Reynolds numbers. By incorporating more of these inclined sections throughout the fin array (e.g. a $\mathrm{W}$-shaped fin structure compared to the current V-shaped structure), the flow could be made unsteady in a larger part of the fin array at low Reynolds numbers, which could enhance performance. This idea should be further studied and it seems to be in commercial use already: T'Joen et al. [13] recently reported on a complex louvered fin design which consists of a W shaped interrupted fin with large inclined sections.

\subsection{Comparison with other fin types}

To quantify the 'performance' of a fin design and provide a fair comparison to other designs, so called 'performance evaluation criteria' or PEC are used. A large number of different criteria exist, e.g. the surface and volume goodness as defined by Kays and London [33]. Here the surface goodness (j/f) will be used as a measure, but it should be stressed that this PEC is just one of many ways to consider the benefits of a fin design. Depending on the actual application the PEC could have an entirely different form, incorporating e.g. manufacturing costs, volume, weight... Figure 12 shows the PEC values for configurations 1 - 9 and compare them to experimental data for three louvered fin designs (DeJong and Jacobi [18]) and one slit fin design (DeJong and Jacobi [17]). This reference data was determined 
experimentally through naphthalene sublimation experiments on scaled models. They are one of the few studies reporting data for the interrupted element only and thus provide a comparison basis as in this study the interrupted element is also studied on its own, without the remainder of the heat exchanger. It should be stressed that the slit fin design presented here has a very small fin pitch ratio and thus a large pressure drop, resulting in lower PEC values. The louver fin designs have louver angle values which are comparable to the high end range of the inclined louvered fin dataset and fin pitch ratios which are comparable to the lower end of the dataset. Increasing the fin pitch ratio or reducing the louver angle will lower the fin efficiency (Zhang and Tafti [22]) making the flow less aligned to the louvers and lowering heat transfer. So within the considered parameter range the louvered fin cases in Fig. 12 represent solid reference cases. It was however the only data of this type available in open literature. Figure 12 suggests that the inclined louvered fin could provide benefits in the considered $\mathrm{Re}_{\mathrm{Lp}}$ range. In particular high fin pitch configurations with a low fin angle (at very low $\mathrm{Re}_{\mathrm{Lp}}$ ) or with an intermediate fin angle (at higher $\mathrm{Re}_{\mathrm{Lp}}$ ) show the highest promise. Configurations with a large fin angle result in low PEC values due to the large pressure drop.

\section{Conclusions}

In this study the flow behaviour and thermo-hydraulic characteristics of a hybrid fin design, the inclined louvered fin, have been reported. Using a combination of experimental (water tunnel visualization) and numerical (CFD simulation) data the flow behaviour was quantified using the fin angle alignment factor $\zeta$. At low Reynolds numbers the flow is deflected due to boundary layer growth. Using wind tunnel experiments local heat transfer coefficients and pressure drop were measured. Comparison of the $\zeta$ curves with the $j$ and $f$ data set out against $\operatorname{Re}_{\mathrm{Lp}}$, revealed the strong link between the flow and $\mathrm{j}-\mathrm{f}$. A sharp decrease in $\zeta$ results in a rise of both $j$ and $f$, as the flow becomes better aligned with the louvers. The onset of unsteady flow further increases $\mathrm{j}$ and $\mathrm{f}$. During the visualization it was shown that unsteady flow occurs at low Reynolds numbers ( 200-300) and that this transition is geometrically driven by recirculation zones on the inclined sections of the fin array. Using the surface goodness PEC it was shown that compared to louvered and slit fins, the inclined louvered fin can offer benefits at low $\mathrm{Re}_{\mathrm{Lp}}$.

\section{Acknowledgments}

The authors would like to express gratitude to the BOF fund (B/05864/01 IV 1) of the Ghent UniversityUGent, which provides funding for the current research program; and to the FWO (Flemish Fund for Scientific Research) for providing support for the stay abroad (V 4/205B MW 5411). The dedication of technicians R. Gillis and P. De Pue is well appreciated.

\section{References}

[1] R.K. Shah, D.P. Sekulic, Fundamentals of Heat Exchanger Design, John Wiley \& Sons, Inc., Hoboken, New Jersey, 2003. 
[2] A. E. Bergles, ExHFT for fourth generation heat transfer technology, Experimental Thermal and Fluid Science 26 (2002) 335-344.

[3] R. Romero-Méndez, M. Sen, K. T. Yang, and R. McCclain, Effect of fin spacing on convection in a plate fin and tube heat exchanger, International Journal of Heat and Mass Transfer 43 (2000), 39-51.

[4] MS. Mon, U. Gross, Numerical study of fin-spacing effects in annular-finned tube heat exchangers, International Journal of Heat and Mass Transfer 47 (2004), 1953-1964.

[5] R. Sedney, A survey of the effects of small protuberances on boundary-layer flows, AIAA Journal 11 (1973), 782-792.

[6] A.M. Jacobi, R.K., Shah, R.K., Heat Transfer Surface Enhancement through the Use of Longitudinal Vortices - a review of recent progress, Experimental Thermal and Fluid Science 11 (1995) 295-309.

[7] A. Joardar, A. M. Jacobi, Heat transfer enhancement by winglet type vortex generator arrays in compact plain-fin-and-tube heat exchangers, International Journal of Refrigeration 31(2008), 87-97.

[8] J. E. O’Brien, M. S. Sohal, Heat transfer enhancement for finned-tube heat exchangers with winglets. Journal of Heat Transfer-Transactions of the ASME 127 (2005), 171-178.

[9] H. Ge, A. M. Jacobi, J.C. Dutton, Air side heat transfer enhancement in offset-strip fin array using delta wing vortex generators - ACRC TR-205, Technical report, ACRC - University of Illinois, 2005.

[10] A. Joardar, A. M. Jacobi, Impact of leading edge delta-wing vortex generators on the thermal performance of a flat tube, louvered-fin compact heat exchanger, International Journal of Heat and Mass Transfer 48 (2005), 1480-1493.

[11] H. M. Metwally, R. M. Manglik, Enhanced heat transfer due to curvature-induced lateral vortices in laminar flows in sinusoidal corrugated-plate channels, International Journal of Heat and Mass Transfer 47 (2004), 2283-2292.

[12] T. A. Rush, T. A. Newell, A. M. Jacobi, An experimental study of flow and heat transfer in sinusoidal wavy passages, International Journal of Heat and Mass Transfer 42 (1999), 1541-1553.

[13] C. T' Joen, H.J. Steeman, A. Willockx, M. De Paepe, Determination of Heat Transfer and Friction Characteristics of an Adapted Inclined Louvered Fin, Experimental Thermal and Fluid Science 30 (2006) 319-327.

[14] R. M. Manglik, A. E. Bergles, Heat-Transfer and Pressure-Drop Correlations for the Rectangular Offset Strip Fin Compact Heat-Exchanger, Experimental Thermal and Fluid Science 10 (1995), 171-180

[15] C. C. Wang, W. S. Lee, W. J. Sheu, A comparative study of compact enhanced fin-and-tube heat exchangers, International Journal of Heat and Mass Transfer 44 (2001), 3565-3573.

[16] C. C.Wang, C. J. Lee, C. T. Chang, S. P. Lin, Heat transfer and friction correlation for compact louvered fin-and-tube heat exchangers, International Journal of Heat and Mass Transfer 42 (1999), 19451956.

[17] N. C. DeJong, A. M. Jacobi, An experimental study of flow and heat transfer in parallel-plate arrays: Local, row-by-row and surface average behavior, International Journal of Heat and Mass Transfer, 40 (1997), 1365-1378. 
[18] N. C. DeJong, A. M. Jacobi, Localized flow and heat transfer interactions in louvered-fin arrays, International Journal of Heat and Mass Transfer 46 (2003), 443-455.

[19] D. K. Tafti, X. Zhang, Geometry effects on flow transition in multilouvered fins - onset, propagation, and characteristic frequencies, International Journal of Heat and Mass Transfer 44 (2001), 4195-4210.

[20] J. Cui, D. K. Tafti, Computations of flow and heat transfer in a threedimensional multilouvered fin geometry, International Journal of Heat and Mass Transfer 45 (2002), 5007-5023.

[21] A. Achaichia, T.A. Cowell, Heat transfer and pressure drop characteristics of flat tube and louvered plate fin surfaces, Experimental Thermal and Fluid Science 1 (1988), 147-157.

[22] X. Zhang, D.K. Tafti, Flow efficiency in multi-louvered fins, International Journal of Heat and Mass Transfer 46 (2003), 1737-1750.

[23] A.C. Lyman, R.A. Stephan, K.A. Thole, L.W. Zhang, S.B. Memory, Scaling of heat transfer coefficients along louvered fins, Experimental Thermal and Fluid Science 26 (2002) 547-563.

[24] T. Tanaka, M. Itoh, M. Kudoh, A. Tomita, Improvement of compact heat exchangers with inclined louvered fins, Bulletin of JSME 27 (1984) 219-226.

[25] A.M. Jacobi, R.K. Shah, Air side flow and heat transfer in compact heat exchangers: a discussion of enhancement mechanisms, Heat Transfer Engineering 19 (1998) 29-41.

[26] R.K. Shah, M.R. Heikal, B. Thonon, P. Tochon, Progress in the numerical analysis of compact heat exchangers, Advances of heat transfer 34 (2001) 363-443.

[27] C. T'Joen, A. Willockx, H.J. Steeman, M. De Paepe, Thermo-hydraulic characteristics of inclined louvered fins, in: Proceedings of the $6^{\text {th }}$ International Conference on Enhanced, Compact and UltraCompact Heat Exchangers, 16-21 September 2007, Potsdam, Germany.

[28] N.C. DeJong, A.M. Jacobi, Flow, heat transfer and pressure drop in the near-wall region of louveredfin arrays, Experimental Thermal and Fluid Science 27 (2003) 237-250.

[29] C. T’Joen, A.M. Jacobi, M. De Paepe, Flow visualization in inclined louvered fins, Experimental Thermal and Fluid Science 33 (2009), 664-674.

[30] C. T'Joen, H. Huisseune, A. Willockx, H. Canière, M. De Paepe, Combined experimental and numerical flow field study of inclined louvered fins, Heat Transfer Engineering, (accepted for publication, Vol. 32, no. 2, 2011)

[31] T'Joen, C., Thermo-hydraulic study of inclined louvered fins, Ph.D. thesis, Ghent University-UGent, Belgium, 2008, http://hdl.handle.net/1854/LU-528875.

[32] R. D. Mehta, P. Bradshaw, Design rules for small low speed wind tunnels, The Aeronautical Journal of the Royal Aeronautical Society 73 (1979), 443-449.

[33] W.M. Kays, A.L. London, Compact Heat Exchangers, 3d ed., McGraw-Hill, New York, 1984.

[34] R. K. Shah, A.L. London, Laminar flow forced convection in ducts, Academic Press, New York, 1978.

[35] X. Zhang, D. K. Tafti, Classification and effects of thermal wakes on heat transfer in multilouvered fins, International Journal of Heat and Mass Transfer 44 (2001), 2461-2473. 
[36] R. J. Moffat, What's new in convective heat transfer?, International Journal of Heat and Fluid Flow, 19 (1998), 90-101.

[37] R.J. Moffat, Describing the uncertainties in experimental results, Experimental Thermal and Fluid Science 1 (1988) 3-17.

[38] K. Kadoya, N. Matsunaga, A. Nagashima, Viscosity and Thermal- Conductivity of Dry Air in the Gaseous-Phase," Journal of Physical and Chemical Reference Data 14(4) (1985) 947-970.

[39] M. E. Springer, K. A. Thole, Experimental design for flowfield studies in louvered fins, Experimental Thermal and Fluid Science 18 (1998), 258-269. 


\section{Figure captions}

Figure 1: The inclined louvered fin and its relevant geometric parameters. The flow passes from left to right.

Figure. 2: Comparison of the experimentally and numerically determined local $\zeta$ values set out against the injection point, configuration $5-\mathrm{Re}_{\mathrm{Lp}}=402$.

Figure 3: Averaged $\zeta_{\mathrm{T}}$ values for configurations 1-5 over the studied Reynolds number range. The filled symbols represent validated numerical simulations. The open symbols are purely numerical cases without accompanying experimental data.

Figure 4: Reynolds number of the onset of unsteady flow at three locations in the inclined louvered fin configurations compared with similar data for louvered fins [19].

Figure 5: Ratio of the amplitude of the local velocity signal to the mean value of this signal determined in the wake of the louvers, configuration 4 - various $\operatorname{Re}_{\mathrm{Lp}}$

Figure 6: Schematic view of open air wind tunnel used for the thermo-hydraulic experiments. Frequency controlled drive (2+1) - centrifugal fan (3) - settling chamber with honeycomb (4) - contraction section (5) settling channel (6) - test section (7) - exit channel section (8) - calibrated orifice plate (9)

Figure 7: A: Comparison of experimental thermo-hydraulic Colburn and friction factor data and numerical predictions based on two dimensional and three dimensional simulations, configuration 2. B: comparison of the louver averaged span wise heat transfer coefficient derived from a three dimensional simulation (symbols) to the two dimension values (lines) for 5 different locations, configuration 2, $\operatorname{Re}_{\mathrm{Lp}}=20$. Figure 8: Local heat transfer coefficients $\left(\mathrm{W} / \mathrm{m}^{2} \mathrm{~K}\right)$ on the different louvers, configuration $8, \mathrm{Re}_{\mathrm{Lp}}=185$. Open symbols: upstream louvers L1 - L5, filled symbols: downstream louvers: L6 - L10. Symbol sequence following the flow direction: $\square, \nabla, \Delta, \circ, \diamond$.

Figure 9: A: Local velocity magnitude $[\mathrm{m} / \mathrm{s}]$ and streamlines around $\mathrm{L} 3, \mathrm{~B}$ : local temperature profile $\left[{ }^{\circ} \mathrm{C}\right]$ around L3 (configuration $1, \mathrm{Re}_{\mathrm{Lp}}=175$ )

Figure 10: Louver averaged heat transfer coefficients (2D CFD results), configuration 2, various $\operatorname{Re}_{\mathrm{Lp}}$. Figure 11: Overview of the Colburn j-factor (A), friction factor (B) and fin angle alignment factor $\zeta_{\mathrm{T}}(\mathrm{C})$ for different configurations. Open symbols: $\varphi=12.64^{\circ}$ ( $\circ$ : config. $2, \nabla$ : config. 1 , $\square$ : config. 8 ), grey symbols: $\varphi=19.78^{\circ}\left(\circ\right.$ : config. 5 , $\nabla$ : config. 7 , $\square$ : config. 6 ), black symbols: $\varphi=30.96^{\circ}(\circ$ : config. $4, \nabla$ : config. 3, $\square$ : config. 9).

Figure 12: Comparison of the surface goodness of configuration 1-9 to three louvered fins (DeJong and Jacobi [18]) and one slit fin (DeJong and Jacobi [17]) 


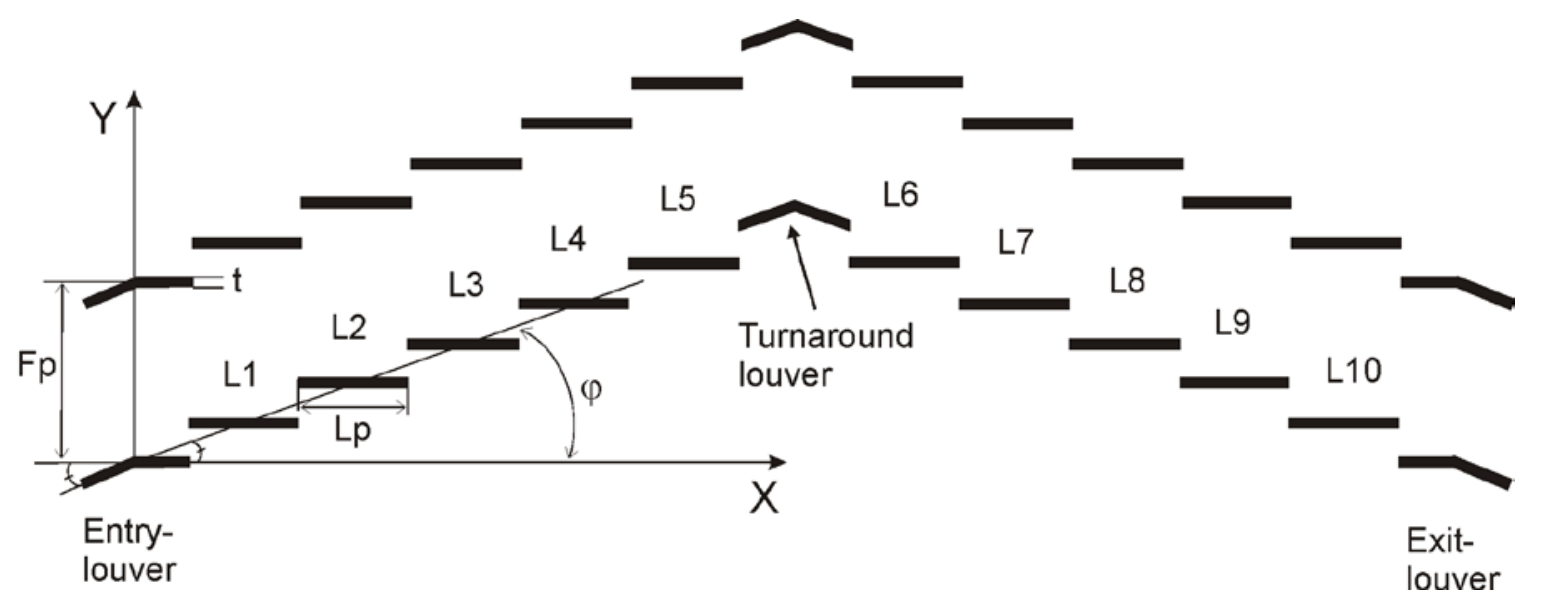

Figure 1: The inclined louvered fin and its relevant geometric parameters. The flow passes from left to right. 


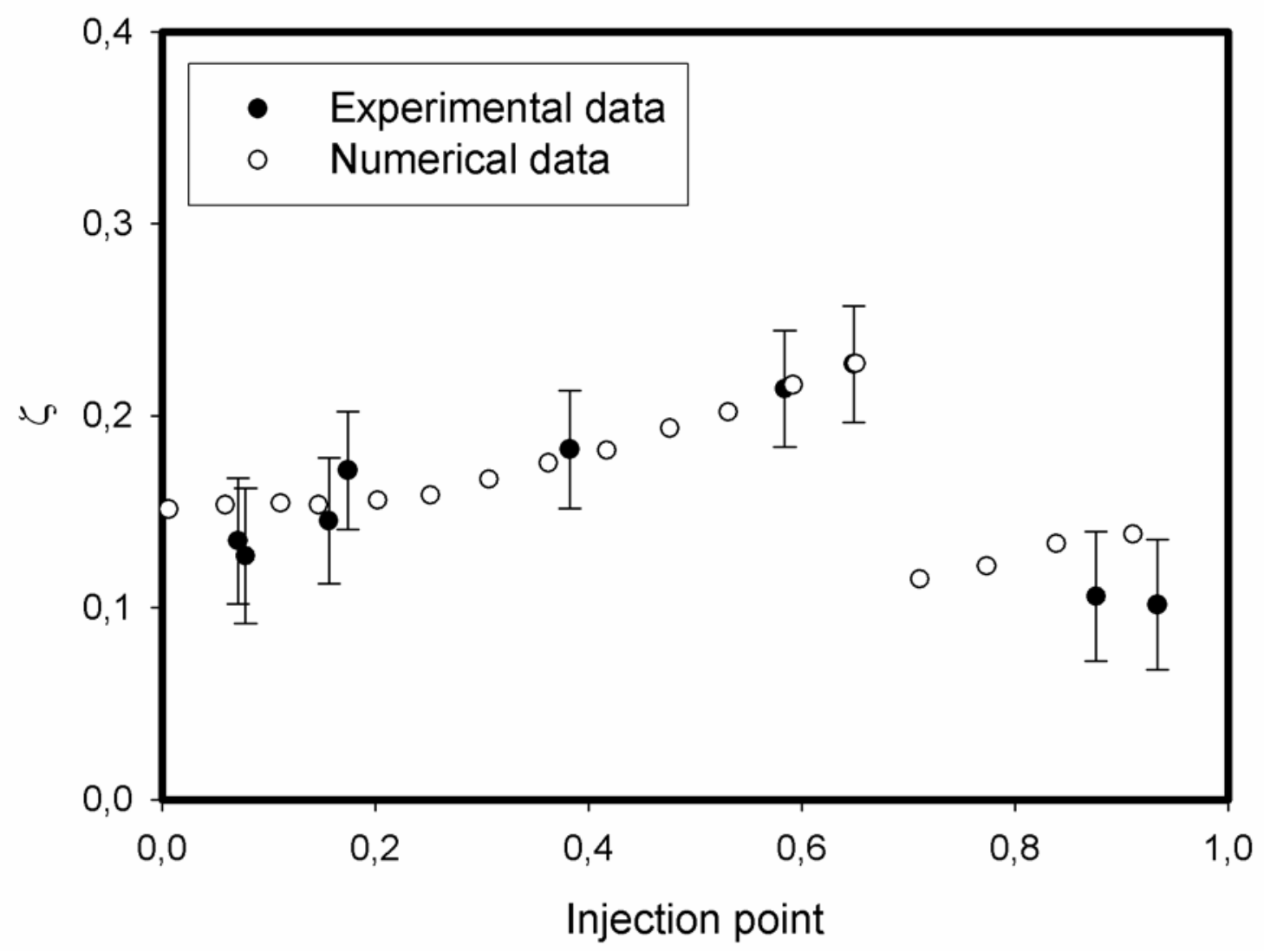

Figure 2: Comparison of the experimentally and numerically determined local $\zeta$ values set out against the injection point, configuration $5-\operatorname{Re}_{\mathrm{Lp}}=402$. 


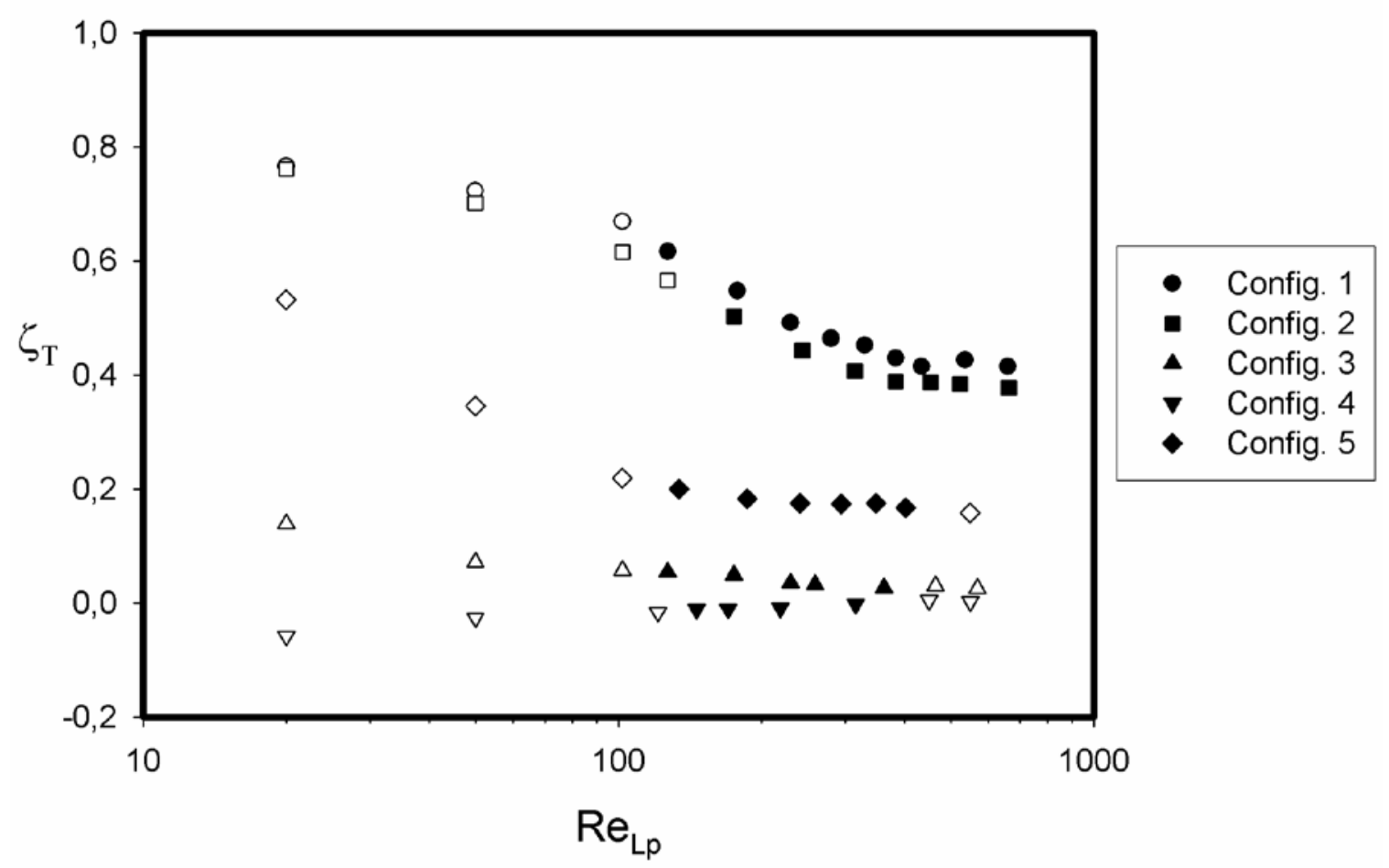

Figure 3: Averaged $\zeta_{\mathrm{T}}$ values for configurations 1-5 over the studied Reynolds number range. The filled symbols represent validated numerical simulations. The open symbols are purely numerical cases without accompanying experimental data. 


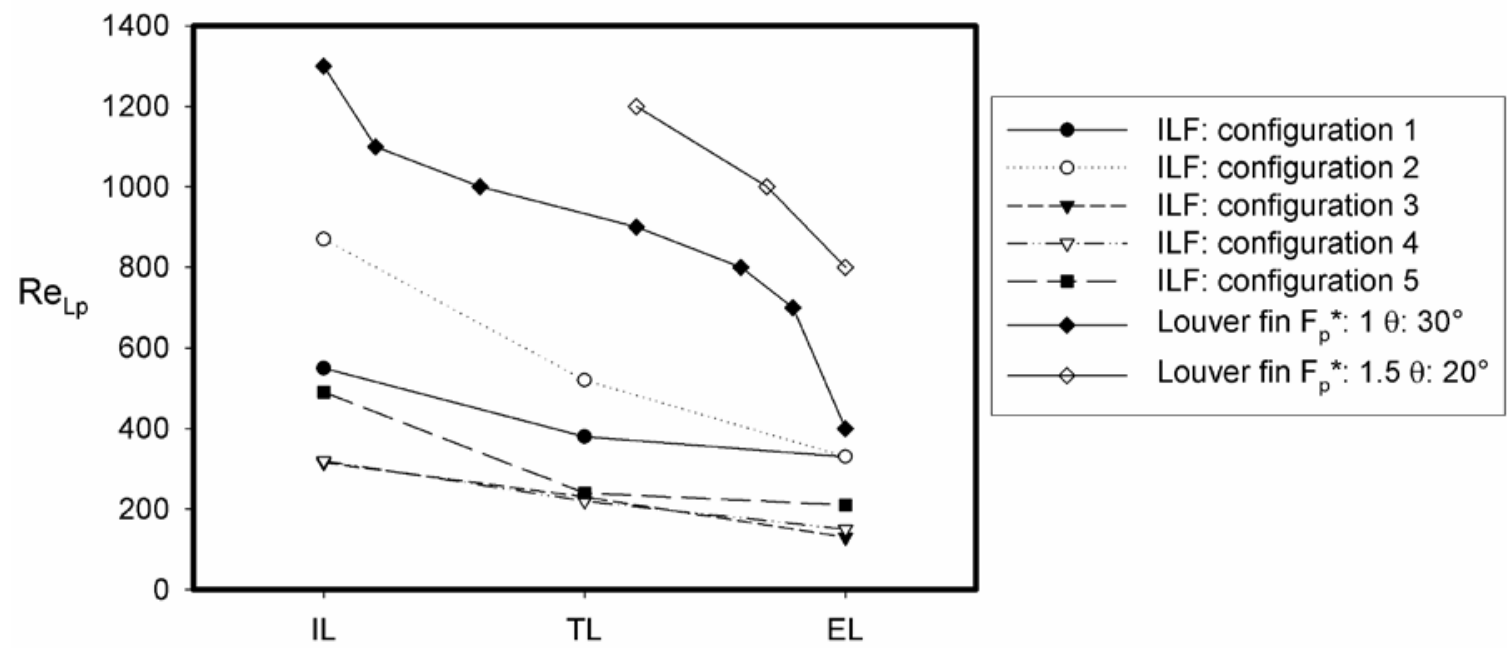

Figure 4: Reynolds number of the onset of unsteady flow at three locations in the inclined louvered fin configurations compared with similar data for louvered fins [19]. 


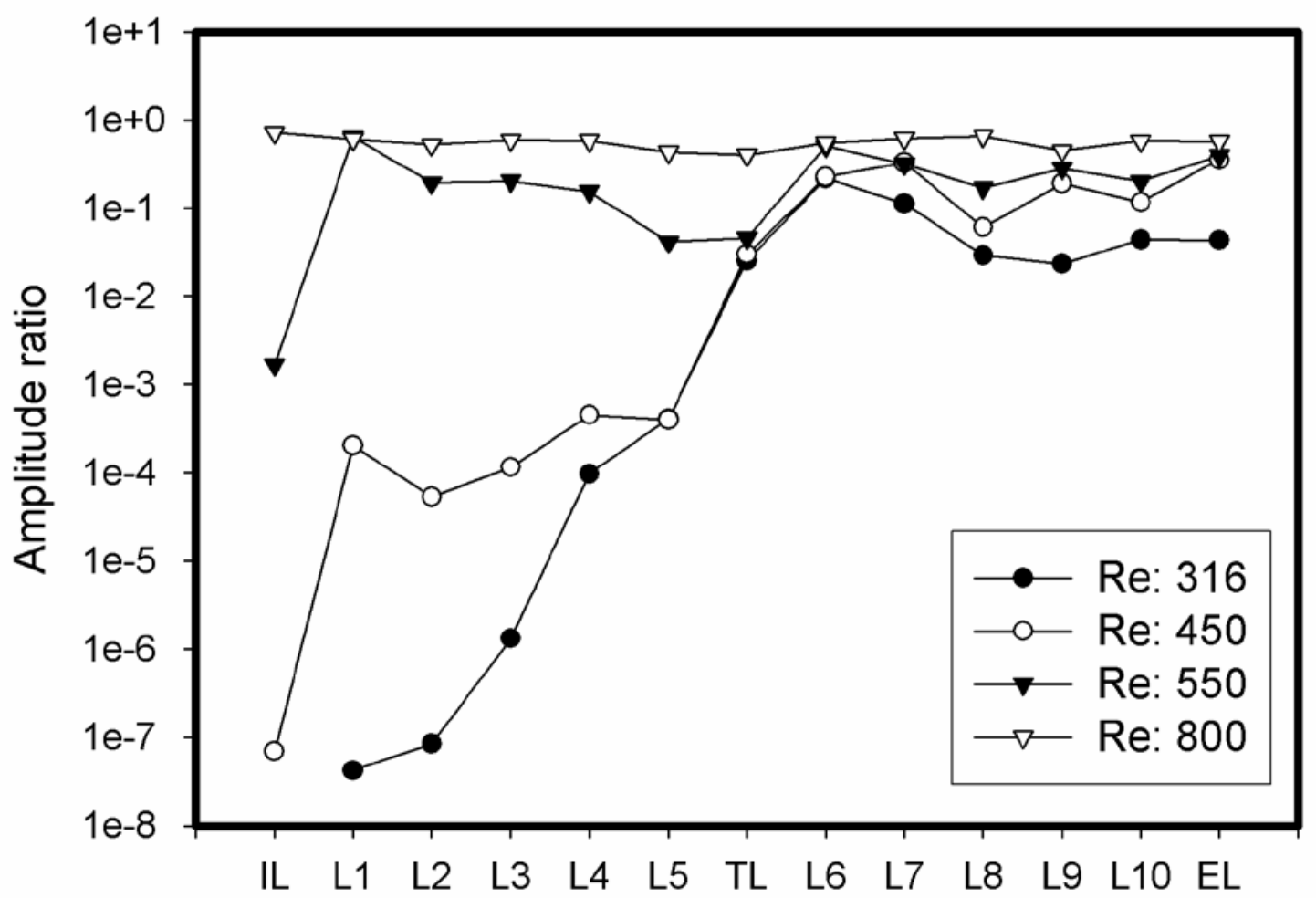

Figure 5: Ratio of the amplitude of the local velocity signal to the mean value of this signal determined in the wake of the louvers, configuration 4 - various $\operatorname{Re}_{\mathrm{Lp}}$ 


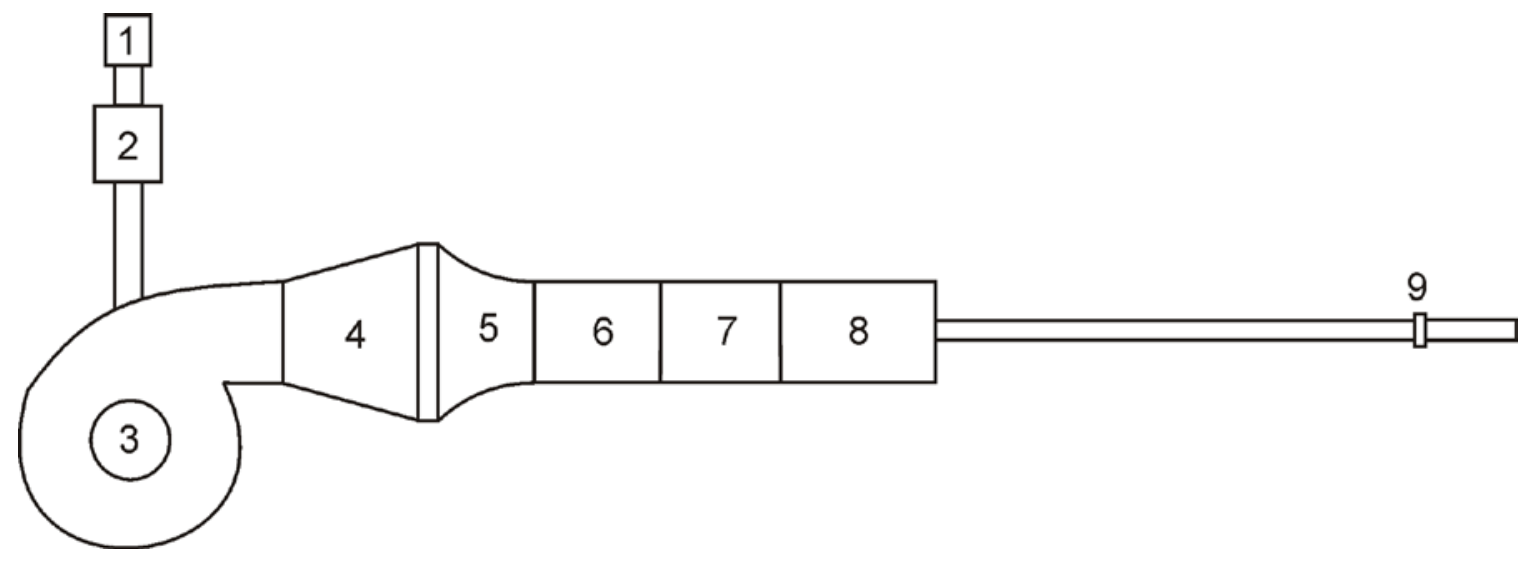

Figure 6: Schematic view of open air wind tunnel used for the thermo-hydraulic experiments. Frequency controlled drive (2+1) - centrifugal fan (3) - settling chamber with honeycomb (4) - contraction section (5) settling channel (6) - test section (7) - exit channel section (8) - calibrated orifice plate (9). 



Figure 7: A: Comparison of experimental thermo-hydraulic Colburn and friction factor data and numerical predictions based on two dimensional and three dimensional simulations, configuration 2. B: comparison of the louver averaged span wise heat transfer coefficient derived from a three dimensional simulation (symbols) to the two dimension values (lines) for 5 different locations, configuration 2, $\operatorname{Re}_{\mathrm{Lp}}=20$. 


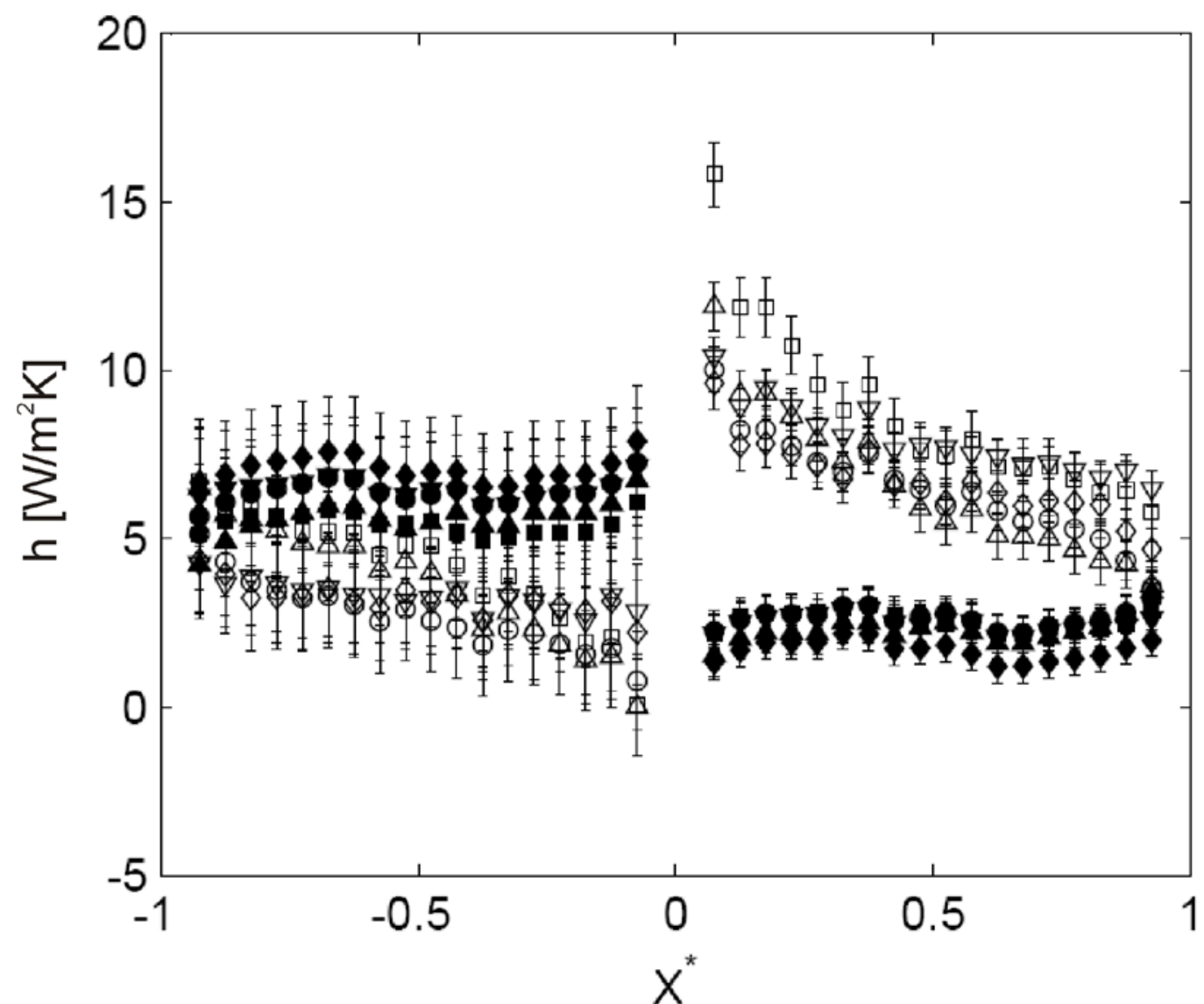

Figure 8: Local heat transfer coefficients $\left(\mathrm{W} / \mathrm{m}^{2} \mathrm{~K}\right)$ on the different louvers, configuration $1, \operatorname{Re}_{\mathrm{Lp}}=185$.

Open symbols: upstream louvers L1 - L5, filled symbols: downstream louvers: L6 - L10. Symbol sequence following the flow direction: $\square, \nabla, \Delta, \diamond, \diamond$. 

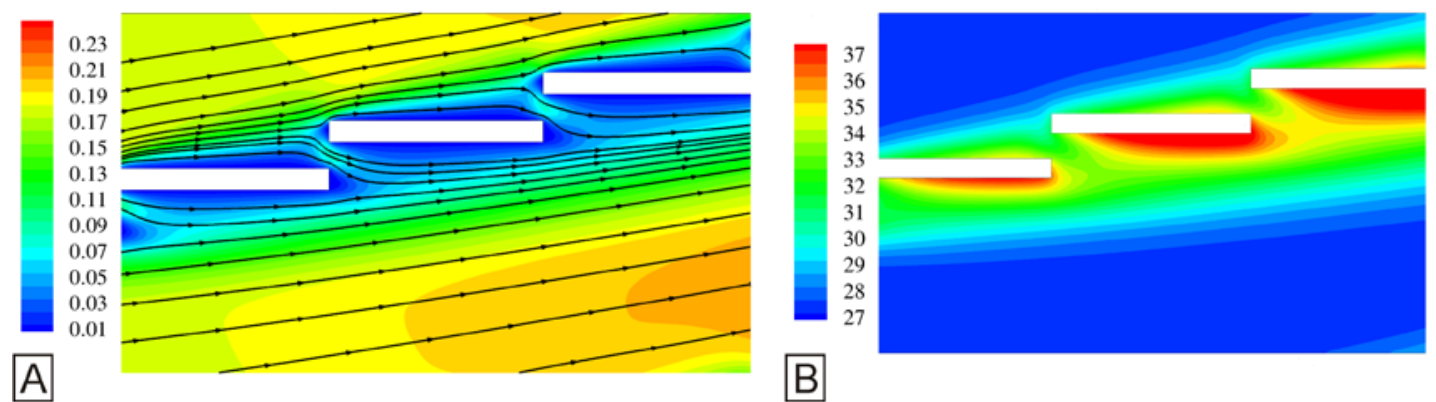

Fig. 9. A: Local velocity magnitude $[\mathrm{m} / \mathrm{s}]$ and streamlines around $\mathrm{L} 3$, B: local temperature profile $\left[{ }^{\circ} \mathrm{C}\right]$ around L3 (configuration $1, \mathrm{Re}_{\mathrm{Lp}}=175$ ) 


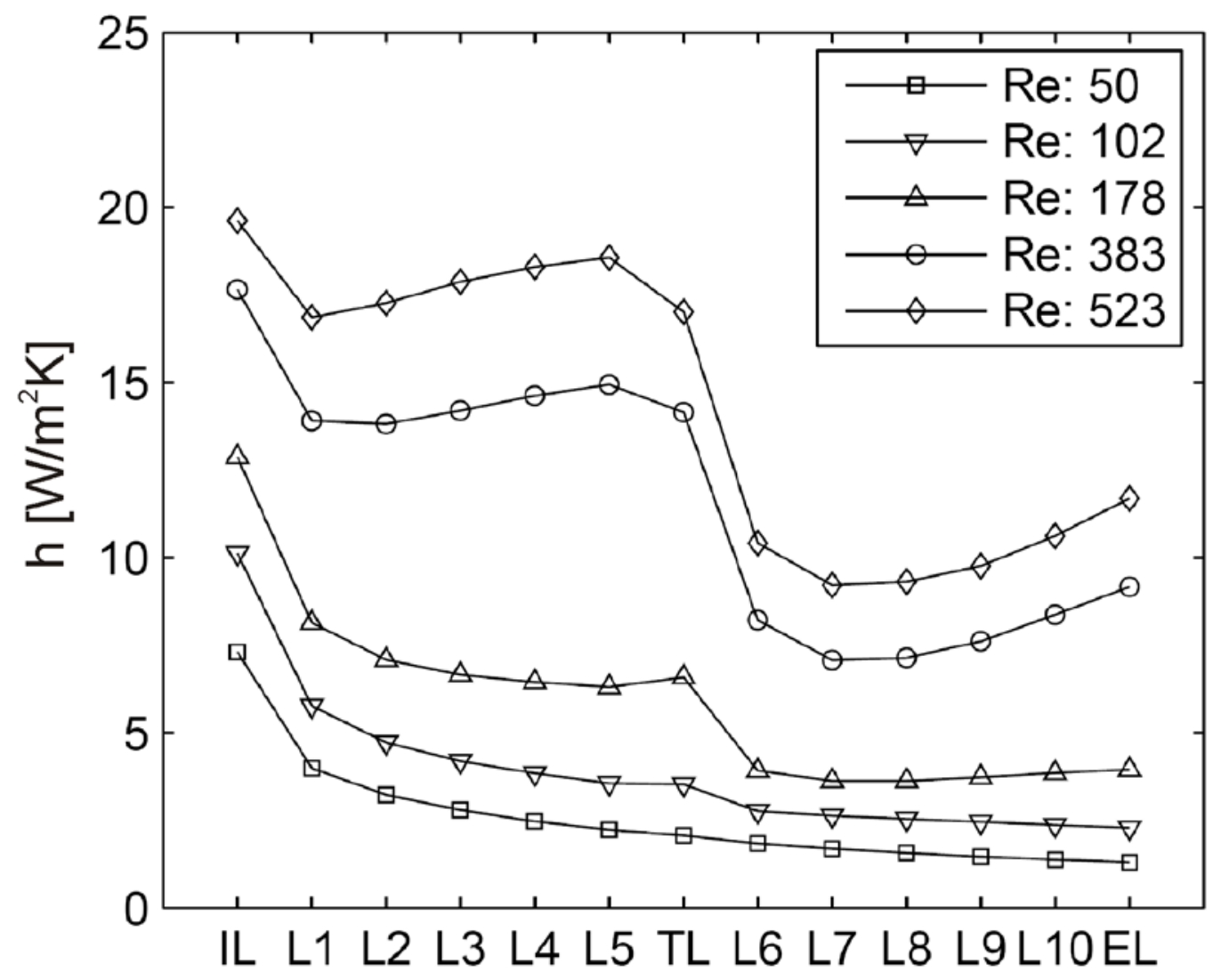

Figure 10: Louver averaged heat transfer coefficients (2D CFD results), configuration 2, various $\operatorname{Re}_{\mathrm{Lp}}$. 

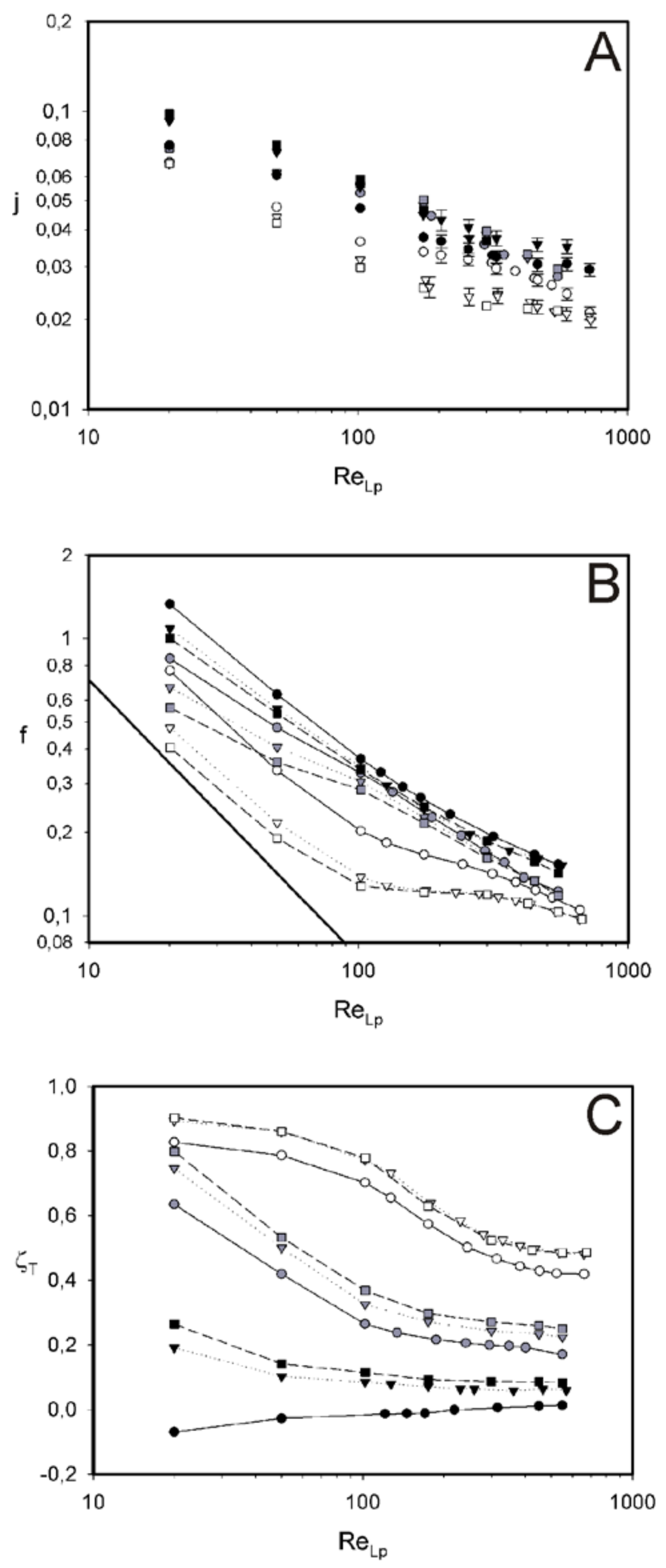

Figure 11: Overview of the Colburn $\mathrm{j}$-factor (A), friction factor (B) and fin angle alignment factor $\zeta_{\mathrm{T}}(\mathrm{C})$ for different configurations. Open symbols: $\varphi=12.64^{\circ}$ ( $\circ$ : config. $2, \nabla$ : config. 1 , $\square$ : config. 8 ), grey symbols: $\varphi=19.78^{\circ}(\circ$ : config. $5, \nabla$ : config. 7 , $\square$ : config. 6$)$, black symbols: $\varphi=30.96^{\circ}(\circ$ : config. $4, \nabla$ : config. 3, $\square$ : config. 9). 


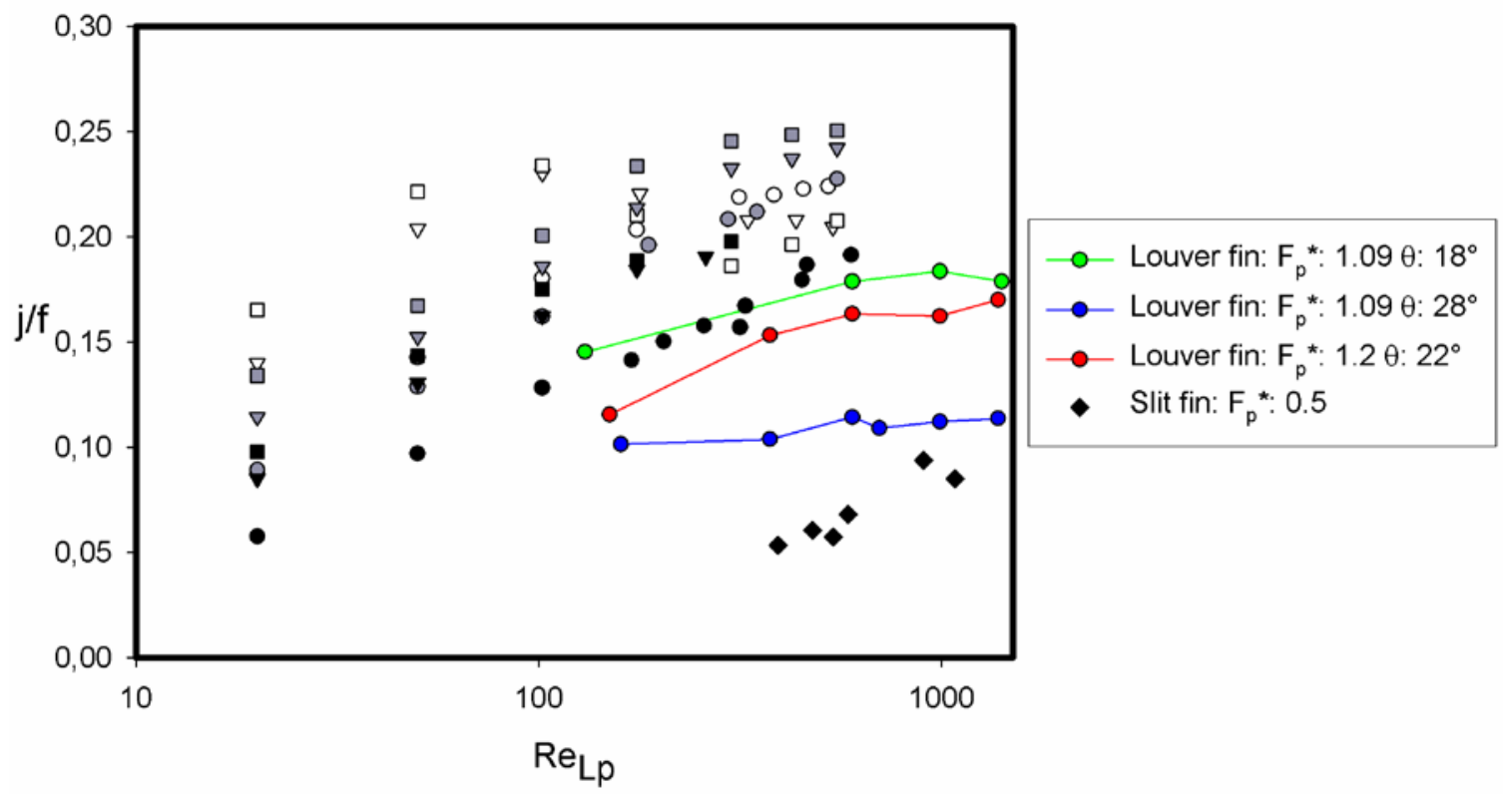

Figure 12: Comparison of the surface goodness of configuration 1-9 to three louvered fins (DeJong and Jacobi [18]) and one slit fin (DeJong and Jacobi [17]) 
Table 1. Geometric parameters of the studied inclined louvered fins

\begin{tabular}{|c|c|c|}
\hline Fin parameters & $\mathrm{F}_{\mathrm{p}}[\mathrm{m}]$ & $\varphi$ \\
\hline Configuration 1 & 0.0340 & $12.64^{\circ}$ \\
Configuration 2 & 0.0225 & $12.64^{\circ}$ \\
Configuration 3 & 0.0340 & $30.96^{\circ}$ \\
Configuration 4 & 0.0225 & $30.96^{\circ}$ \\
Configuration 5 & 0.0276 & $19.78^{\circ}$ \\
Configuration 6 & 0.0400 & $19.78^{\circ}$ \\
Configuration 7 & 0.0340 & $19.78^{\circ}$ \\
Configuration 8 & 0.0400 & $12.64^{\circ}$ \\
Configuration 9 & 0.0400 & $30.96^{\circ}$ \\
Configuration 10 & 0.0225 & $19.78^{\circ}$ \\
Configuration 11 & 0.0300 & $12.64^{\circ}$ \\
Configuration 12 & 0.0300 & $19.78^{\circ}$ \\
Configuration 13 & 0.0300 & $30.96^{\circ}$ \\
\hline
\end{tabular}

